\title{
Substantiation of a New Calculation and Selection Algorithm of Optimal Heat Exchangers with Nanofluid Heat Carriers Taking into Account Surface Forces
}

\author{
Yuriy Bilonoga ${ }^{1 *}$, Volodymyr Stybel$^{2}$, Oksana Maksysko $^{1}$, Uliana Drachuk ${ }^{1}$ \\ ${ }^{1}$ Faculty of Food Technologies and Biotechnology, Stepan Gzytsky Natoinal University of Veterinary Medicine and \\ Biotechnologies Lviv, 50 Pekarska, Lviv 79010, Ukraine \\ ${ }^{2}$ Faculty of Veterinary Medicine, Stepan Gzytsky Natoinal University of Veterinary Medicine and Biotechnologies Lviv, 50 \\ Pekarska, Lviv 79010, Ukraine
}

Corresponding Author Email: yuriy_bilonoha@ukr.net

https://doi.org/10.18280/ijht.390602

Received: 12 July 2021

Accepted: 26 November 2021

\section{Keywords:}

$B l$ and Bl turb. numbers, heat exchangers, thermal conductivity turbulent, viscosity turbulent, surface tension coefficient, nanofluids

\begin{abstract}
The article examines the problem of correct, accurate calculation and optimization of the choice of heat exchange equipment when using nanofluid heat carriers for heat treatment of liquid food products using milk as an example. To solve this problem, the motion of a metal nanoparticle in a turbulent flow of the main coolant was simulated using the methods of similarity theory, taking into account the action of surface forces in the laminar boundary layer. New formulas are obtained for a qualitative assessment of the average thickness of the laminar boundary layer that appears around a turbulently moving metal nanoparticle. A number of qualitative correlations with other literature sources that explain the behavior of nanoparticles in a turbulent liquid medium are shown. A new approach to heat transfer processes is considered taking into account the theory of $\mathrm{J}$. Boussinesq, which gives an idea of turbulent viscosity and thermal conductivity, as well as a comparison of the resistance forces to the surface forces. The physical meaning of the previously obtained by us new similarity numbers $B l$ and $B l_{t u r b}$. and their application in our new numerical equation for calculating heat exchangers is considered, and a new express method for evaluating the efficiency of using nanofluid heat carriers is proposed. The proposed method of express calculation shows that the mixture $\mathrm{H}_{2} \mathrm{O}+\mathrm{EG}$ (60:40) improves the heat exchange properties of water by $+12.86 \%$, and the mixture $\left(\mathrm{H}_{2} \mathrm{O}+\mathrm{EG}(60: 40)+1.5 \% \mathrm{TiO}_{2}\right)$ and (milk $+0.5 \%$ pumpkin seed oil) - by $+16.75 \%$, which corresponds to the experiments and our calculations, and the well-known express method based on classical numerical equations shows a deterioration by $-4.5 \%$ and, accordingly, by $-1.2 \%$.
\end{abstract}

\section{INTRODUCTION}

It is shown in the work [1] that the maximum and minimum temperatures and velocities in a turbulent flow of liquids and gases are related by the relation (1). In formula (1), the recommended average ratio of the momentum $A \tau$ and heat transfer coefficients $A q$ in the middle of the flow is about 0.769 [2].

$$
\frac{T_{\min .}}{T_{\max .}}=\left(\frac{V_{\min .}}{V_{\max .}}\right)^{A \tau / A q}
$$

It should be emphasized that $\mathrm{H}$. Reichardt did not rely on to any theory of turbulence, and he drew conclusions only on the basis of his experimental data. After these studies, many theoretical and experimental works were published on the laws of the distribution of velosities and temperatures in liquid and gas flows, which are compactly collected in the H. Schlichting monograph and which confirm dependence (1) (see pages) [2].
1.1 Some examples of classical numerical equations for calculating heat transfer coefficients when using nanofluids

Eq. (2) is a classic numerical equation that calculates heat transfer coefficients for different types of heat exchangers and different liquid and gas heat transfer fluids.

$$
N u=B(\operatorname{Re})^{X} \cdot(\operatorname{Pr})^{Y}
$$

Constant $B$ and indicators $X, Y$ are found experimentally and are in no way interconnected. We present two well-known classical equations of turbulent motion of a coolant in pipes and spaces of shell-and-tube (a, b) and plate (c) heat exchangers (3) [3]:
a) $N u=0.023 \operatorname{Re}^{0.8} \operatorname{Pr}^{0.43}$
b) $N u=0.24 R e^{0.6} \operatorname{Pr}^{0.36}$
c) $N u=0.135 \operatorname{Re}^{0.73} \operatorname{Pr}^{0.33}$;

Constants $B$ and exponents $X, Y$ are purely empirical, which, when using various nanofluids, completely change their scalar values and cannot be predicted analytically for quick 
engineering calculations. In addition, it should be emphasized that initially the structures of equations of the type (3) were not obtained by a universal method of dimensional analysis. They were purely empirical.

In the work of Pak and Cho [4] it was proposed to improve the classical Eq. (3) for the theoretical calculation of the convection coefficient when using a nanofluid with $\mathrm{TiO}_{2}$ nanoparticles by changing the exponent at the Prandl number from 0.43 to 0.5 .

In the work of Maiga et al. [5], where $\mathrm{Al}_{2} \mathrm{O}_{3} /$ water nanofluids were used, the exponents were changed at both Reynolds and Prandtl number, as well as the constant $B$, and Eq. (5) was proposed (Table 1).

Eqns. (6-8) by Petukhov [6], Gnielinski [7] and Buongiorno [8] which are also used to calculate nanofluids, differ slightly in structure from (3), but contain the same classical numbers Nusselt, Reynolds and Prandtl in fractional degree corrected for the coefficient of friction $f$ (Table 1).

Equations by Xuan and Li (9) [9], Duangthongsuk and Wongwises (10) [10], Vajjha et al. (11) [11], Godson Asirvatham et al. (12) [12] (Table 1) in their structure, they are similar to equations of the type (3), however, they have corrections for the fractional and concentration composition of specific nanofluids. In this case, the constants B and the exponents $X$ and $Y$ at the Reynolds and Prandtl numbers are completely different. These equations cannot analytically predict the effectiveness of the addition of one or another nanofluid, and when changing the concentration and fractionation in water and other basic heat transfer fluids, they must be experimentally verified and supplemented. This increases the complexity of the experiments. They lose their versatility.

A similar situation with the calculation of plate heat exchangers when using nanofluids (Eqns. 13-15) (Table 2). In Eq. (13) by Elias et al. [13] constants $B$ and exponents $X$ and $Y$ differ from each other by thousandths in different slopes of the chevron angles $\beta$ of the plates. At the same time, it seems to us that this insignificant difference is due to a change in the chevron angle $\beta$, which affects the degree of turbulence of the coolant moving between the plates.

Empirical Eqns. (14 and 15) presented in the works of Elias et al. [14] and Huang et al. [15], respectively, the constants and exponents also differ by hundredths.

Tables 1 and 2 show that as soon as some conditions for the use of nanofluids and combinations of their various parameters change, as well as the heat exchangers themselves, new expensive experiments with new equipment are required. Such calculations using classical empirical equations have lost their versatility and cannot be used for an express assessment of the efficiency of specific nanofluids in specific heat exchange equipment.

Table 1. Convective heat transfer correlations of $\mathrm{TiO}_{2}$ nanofluids for calculating shell-and-tube heat exchangers

\begin{tabular}{|c|c|}
\hline Numerical equation & Relevant information \\
\hline \multirow{5}{*}{$\begin{array}{l}N u=0.021 \cdot \operatorname{Re}^{0.8} \operatorname{Pr}^{0.5} \\
\text { Pak and Cho, (1998) }[4]\end{array}$} & Experimental study \\
\hline & Turbulent flow $\mathrm{Al}_{2} \mathrm{O}_{3} /$ water nanofluids $\mathrm{TiO}_{2} /$ water nanofluids: \\
\hline & $(0.0 \leq \phi \leq 3.0)$ \\
\hline & $\left(10^{4} \leq \operatorname{Re} \leq 10^{5}\right)$ \\
\hline & $(6.5 \leq \operatorname{Pr} \leq 12.3)$ \\
\hline \multirow{6}{*}{$N u=\frac{(0.125 f) \operatorname{Re} \cdot \operatorname{Pr}}{1.07+12.7(0.125 f)^{0.5}\left(\operatorname{Pr}^{2 / 3}-1\right)} f=(0.79 \ln R e-1.64)^{-2}$} & $\begin{array}{l}\text { Numerical study Turbulent flow } \mathrm{Al}_{2} \mathrm{O}_{3} / \text { water nanofluids } \\
\qquad(0.0 \leq \phi \leq 10.0)\end{array}$ \\
\hline & $\left(10^{4} \leq \operatorname{Re} \leq 5.10^{5}\right)$ \\
\hline & $(6.6 \leq \operatorname{Pr} \leq 13.9)$ \\
\hline & Numerical study \\
\hline & Fully developed turbulent flow: \\
\hline & $\left(5 \cdot 10^{5} \leq \operatorname{Re} \leq 5 \cdot 10^{6}\right)$ \\
\hline Petukhov (1970) [6] & $(0.5 \leq \operatorname{Pr} \leq 2000)$ \\
\hline \multirow{2}{*}{$N u=\frac{(0.125 f)(\operatorname{Re}-1000) \operatorname{Pr}}{1+12.7(0.125 f)^{0.5}\left(\operatorname{Pr}^{2 / 3}-1\right)}$} & Numerical study \\
\hline & Fully developed turbulent flow: \\
\hline$f=(0.79 \ln R e-1.64)^{-2}$ & $\left(3 \cdot 10^{3} \leq \operatorname{Re} \leq 5 \cdot 10^{6}\right)$ \\
\hline \multirow{2}{*}{ Gnielinski (1976) [7] } & $(0.5 \leq \operatorname{Pr} \leq 2000)$ \\
\hline & \\
\hline \multirow{2}{*}{$N u=\frac{(f / 8)(\operatorname{Re}-1000) \operatorname{Pr}}{1+\delta_{\tau} \sqrt{(f / 8)}\left(\operatorname{Pr}^{2 / 3}-1\right)}$} & Numerical study \\
\hline & Fully developed turbulent flow: \\
\hline \multicolumn{2}{|l|}{ Buongiorno (2006) [8] } \\
\hline \multirow{4}{*}{$\begin{array}{c}N u=0.0059\left(1+7.686 \phi^{0.6886}\right) \cdot \operatorname{Re}^{0.9238} \cdot \operatorname{Pr}^{0.4}(9) \\
\text { Xuan and Li, (2003) [9] }\end{array}$} & Experimental study Turbulent flow $\mathrm{Cu}$ /water nanofluids \\
\hline & $\phi$ - volume concentration (\%) $\varphi$ - volume fraction, \\
\hline & $(0.0 \leq \phi \leq 2.0)$ \\
\hline & $\left(10^{4} \leq \operatorname{Re} \leq 2.5 .10^{5}\right)$ \\
\hline \multirow{2}{*}{$\begin{array}{l}\quad N u=0.074 \cdot \operatorname{Re}^{0.707} \operatorname{Pr}^{0.385} \cdot \varphi^{0.074}(10) \\
\text { Duangthongsuk and Wongwises, (2010) [10] }\end{array}$} & $\begin{array}{l}\text { Experimental study Turbulent flow } \mathrm{TiO}_{2} / \text { water nanofluids } \\
\qquad(0.2 \leq \phi \leq 2.0)\end{array}$ \\
\hline & $\left(3 \cdot 10^{3} \leq \operatorname{Re} \leq 1.8 \cdot 10^{4}\right)$ \\
\hline$N u=0.065 \cdot\left(\operatorname{Re}^{0.65}-60.22\right)$ & Experimental study Turbulent flow $\mathrm{Al}_{2} \mathrm{O}_{3} /$ water nanofluids \\
\hline$\left(1+0.0169 \phi^{0.5} \cdot \varphi^{0.074}\right) \operatorname{Pr}^{0.542}$ & $\mathrm{CuO} /$ water nanofluids $\mathrm{SiO}_{2} /$ water nanofluids \\
\hline
\end{tabular}


Experimental study

Godson Asirvatham et al. (2011) [12]

Table 2. Correlations of convective heat transfer of $\mathrm{Al}_{2} \mathrm{O}_{3} /$ water and $\mathrm{SiO}_{2}$ / water nanofluids for calculation of plate heat exchangers

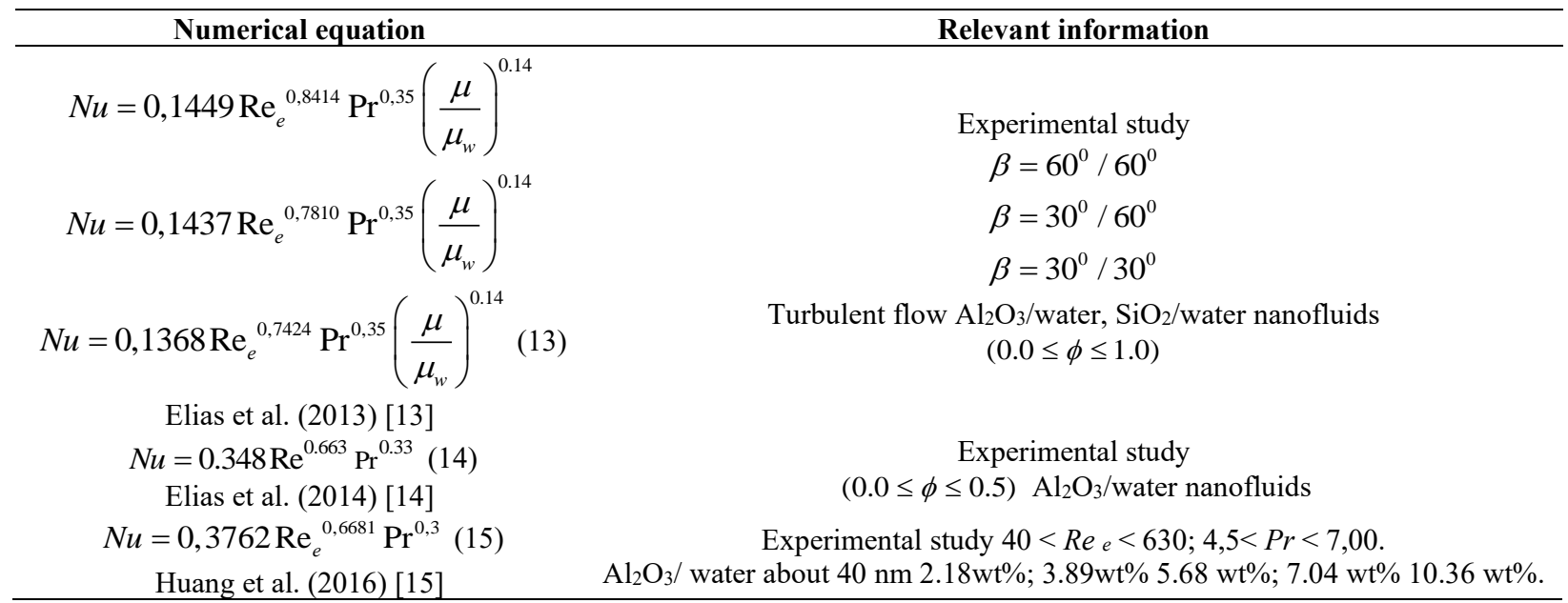

In our work [16], we proposed to move away from the practice of empirical calculations of heat exchangers with nanofluidic coolants using classical numerical equations based on classical similarity numbers, and to propose a new universal one, in which not the fractional and concentration compositions of nanofluids, but their main thermophysical quantities for turbulent coolant flow (turbulent viscosity and thermal conductivity). We also proposed to consider the flow of a coolant with nanofluids taking into account surface forces [16]. We set out the main prerequisites for such an approach below.

It is indicated that to increase the efficiency of using a coolant, it is necessary that the ratio of thermal conductivity to viscosity of nanofluid should increase with the addition of nanoparticles [17]. In addition, in numerous works on heat transfer, it is indicated that the average diameter of nanoparticles significantly affects the efficiency of heat transfer of nanofluid, namely, at relatively large diameters of nanoparticles, the thermal conductivity of the nanofluid increases and its viscosity decreases [17-20]. It was shown in that the main thermophysical characteristics of nanofluids viscosity and thermal conductivity depend on the average diameter of the nanoparticles, as well on the of their material (Figure 1).

It was clearly shown in the study of Timofeeva et al. [18] that the tendency for an increase in the thermal conductivity of nanofluids with an increase in the average diameter of $\mathrm{SiC}$ nanoparticles in the range of $(16-90) \mathrm{nm}$ persists with a change in the base fluid from $\mathrm{EG} / \mathrm{H}_{2} \mathrm{O}$ to $\mathrm{H}_{2} \mathrm{O}$. The base fluid $\mathrm{EG} / \mathrm{H}_{2} \mathrm{O}$ shows a more significant increase in heat transfer properties compared to water $\mathrm{H}_{2} \mathrm{O}$ (Figure 1).

The abscis axis of the diagram shows the average diameters of nanoparticles in the order of their increase, and the ordinate shows the percentage increase in the thermal conductivity of the nanofluid compared to the model liquid - water. These results indicate that the higher heat transfer properties do not belong to water, but to solutions of glycols of various concentrations in water as a base fluid. Further, in sections 3.6.1 - 3.6.3 we are repeatedly sent to this material (Figure 1).

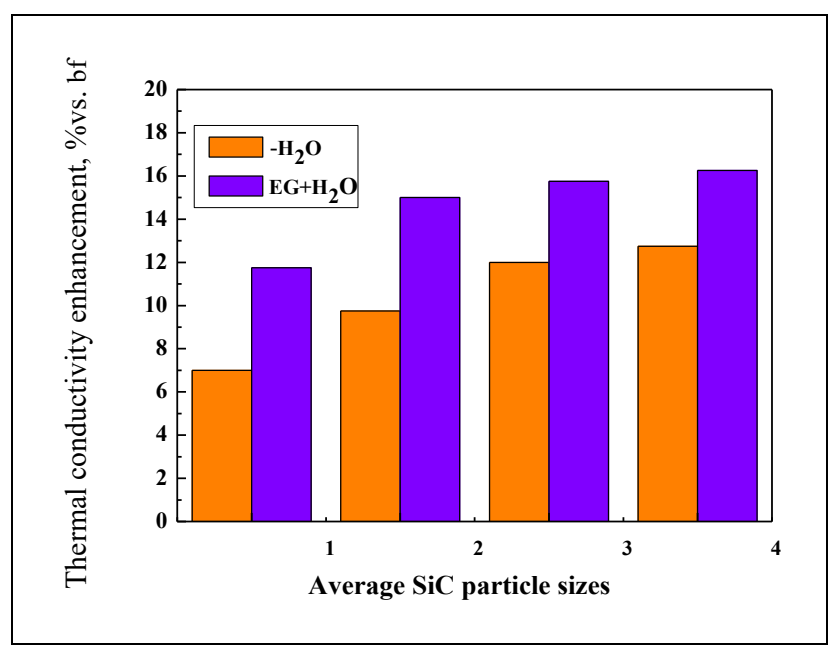

Figure 1. Comparison of the thermal conductivity enhancement in 4 vol.\% SiC nanofluids with $\mathrm{EG} / \mathrm{H}_{2} \mathrm{O}(50: 50)$ and $\mathrm{H}_{2} \mathrm{O}$ as base fluids at various particle sizes: $1-16 \mathrm{~nm} ; 2-$ $28 \mathrm{~nm} ; 3-66 \mathrm{~nm} ; 4-90 \mathrm{~nm}$; [18]

We are approaching the fact that using nanoparticles of certain average diameters and a certain material, it is possible to control the system for transferring the amount of heat in nanofluidic coolants, that is, to select heat nanotechnology.

However, how to analytically predict this - to select the required parameters and thermophysical characteristics of nanoparticles and base fluid without laborious and expensive experiments, using a fast and accurate express method for assessing the efficiency of using a particular nanofluid in a particular heat exchange process? 
At the same time, some researchers insist on the impossibility of calculating the viscosity and thermal conductivity of nanofluid heat carriers included in classical equations of the type (3) by classical methods and formulas $[17,20]$. However, these studies are still based on classical numerical Eq. (3) and take into account the values of molecular viscosity and thermal conductivity of nanofluids under static immobile conditions of heat carriers.

\subsection{Molecular dynamics method (MDM) for studying the behavior of nanoparticles in a liquid medium}

MDM, of course, is the most effective and accurate method in terms of studying the behavior of nanoparticles in a liquid medium. Nanoscale particles suggest that they collide with the molecules of the medium under almost equivalent conditions, that is, they receive an almost equivalent impulse from them.

Considering the works [20-23] in the field of MDM, a of important conclusions selves:

- a feature of the movement of a nanoparticle in a liquid or gas medium is the formation of microfluctuations around it and their interaction with the nanoparticle [21, 22];

- in a liquid medium, molecules appear around the nanoparticle, the speeds of which are comparable with its speed of movement [21, 22];

- near microfluctuations, the nanoparticle velocity relaxes;

- microfluctuations around nanoparticles are characterized by local changes in the concentration of medium molecules;

- behind the movement of the nanoparticle there is a strong rarefaction $[21,22]$;

- in the direction of motion, the process of relaxation of the velocity of the nanoparticle occurs, which is accompanied by an increase in the density of the liquid [21, 22].

The law of motion of a ball in a fluid at the macrolevel determined G.G. Stokes and concluded it in the well-known formula $F=3 \pi d V \mu$. Undoubtedly, the conclusions from [21, $22]$ at the nanolevel correlate with the well-known conclusions of G.G. Stokes at the macrolevel.

Using the methods of molecular dynamics MDM in computer numerical simulation, it was found that the resistance force of the medium to the motion of nanoparticles at the beginning of motion is 2 - 3 times higher than the Stokes force, and then asymptotically approaches the equilibrium value and is approximately equal to the Stokes force for relatively large masses of nanoparticles [23]. After relaxation is completed, the resistance force is completely determined by hydrostatic pressure, and its vector projection is greater than zero for $\theta<90^{\circ}$ and less than zero for $\theta>90^{\circ}$. The force is also anisotropic and decreases sharply with increasing angle $\theta$ [23].

However, unfortunately, it was not indicated what kind of force it is, what its nature is [23]? Based on our previous studies [16, 24-26], we argue that this resistance force of the base fluid to the movement of the nanoparticle is the interfacial surface tension force at the interface between the solid nanoparticle and the fluid, which depends on hydrophilicity (cosine of the contact angle, $\cos 0^{\circ}=1$ and $\cos 90^{\circ}=0$ ), which, in turn, can significantly change its vector and scalar value. Since the action of surface forces is molecular in nature, we argue that this approach has a completely acceptable basis.

\section{PROBLEM FORMULATION}

The well-known classical numerical equations for calculating heat exchange equipment cannot provide reliable calculation and selection of optimal heat exchangers when using nanofluids in chemical, food and other heat technologies, especially at temperatures above $50^{\circ} \mathrm{C}$ [16]. This is due to the fact that these numbers contain the thermophysical characteristics (dynamic viscosity and thermal conductivity) of heat transfer fluids in a static (stationary) state. In addition, surface forces are not taken into account [16, 24-26]. At the same time, all heat exchange equipment is selected and calculated taking into account the turbulent movement of heat carriers. In this work, we set ourselves the following tasks:

- to simulate the behavior of a nanoparticle in a turbulent flow of a coolant taking into account surface forces and show their qualitative predominance in laminar boundary layer (LBL);

- to point out the inaccuracy of the classical methods of calculating heat exchange equipment in chemical, food and other heat technologies when using nanofluids, especially at temperatures above $50^{\circ} \mathrm{C}$, where there is a discrepancy with experiments in the range of $(15-20) \%$ or more;

- to propose a new express method for testing the efficiency of heat transfer agents in heat exchangers using the example of heating milk with hot water with appropriate nanoadditives based on a new numerical equation;

- give an example of calculating a shell-and-tube heat exchanger by a new method and show the advantages of the latter when using nanofluid heat transfer fluids.

\section{SIMULATION OF THE NANOFLUID HEATING AGENTS AND ANALYTICAL EXPRESS- CALCULATION OF HEAT EXCHANGERS}

\subsection{Modeling the motion of solid nanoparticles in a turbulent coolant flow taking into account the similarity theory}

We take into account the experiments and theoretical results presented in the excellent detailed review by Mishra et al. [27], which indicate that the study of the behavior of nanofluids as thermal agents requires new models and new approaches, since classical developments do not fully and insufficiently explain the increase in thermal conductivity in nanofluid systems.

The most realistic, in our opinion, are models that assume the formation of a surface monolayer of a base fluid around a nanoparticle, the physical characteristics of which resemble the properties of the nanoparticle itself. However, to date, there are no theoretical or experimental studies that could predetermine the behavior of this monolayer [27].

Jiang et al. [28] shows that the presence of an interphase layer appearing at the solid (nanoparticle) - liquid interface is one of the main factors in increasing the thermal conductivity of nanofluids. In contrast to other classical models, it takes into account some effects - the volume fraction, thickness and thermal conductivity of the interphase layer, as well as the average size of nanoparticles. According to this model, the solid-like nanolayer acts as a thermal bridge between the schematic cylindrical nanoparticle and the base fluid [28].

Consider a solid nanoparticle that moves chaotically in a heat-transfer fluid in a turbulent $(\mathrm{T})$ regime (Figure 2 ). 


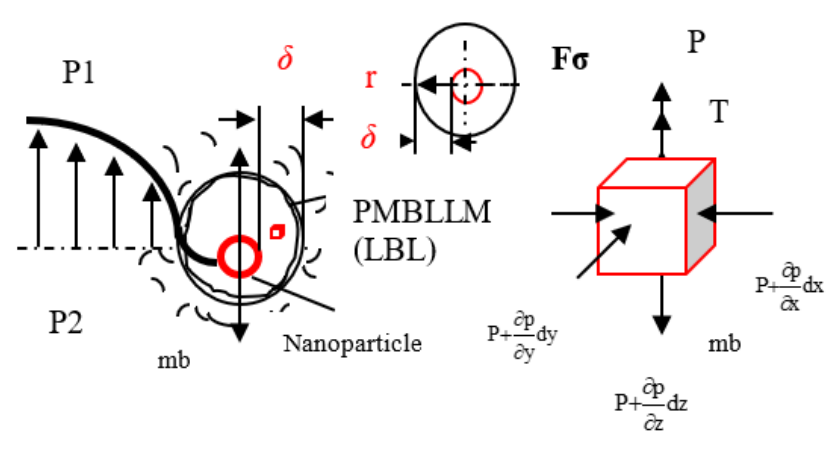

Figure 2. Scheme of PMBLLM (LBL) formation around a mobile nanoparticle in a liquid coolant with a $\mathrm{T}$ - regime

At a certain time, an excess pressure $\mathrm{P}_{2}$ arises in front of the nanoparticle, and behind it, the missing pressure P1 [20-23]. At the same time, a polymolecular boundary layer of liquid molecules (PMBLLM) (LBL) is formed around the nanoparticle by a powerful field of surface forces, the characteristics of which differ from those in the volume. The surface energy of metal surfaces and their oxides in the solid about state is of $-\sigma \approx(0.5-3) \mathrm{J} / \mathrm{m}^{2}$ [29-32], whereas the surface energy of liquids is about $\sigma \approx(20-72) \cdot 10^{-3} \mathrm{~J} / \mathrm{m}^{2}$. The relative gradient of the scalar field of surface energy of a solid - liquid is on of - (25-40) times or more. A nanoparticle in a liquid turbulent medium, colliding with molecules, receives additional chaotic impulses from them. MDM suggest that the behavior of a nanoparticle at the nanolevel correlates with the behavior of a sphere in a liquid at the macrolevel [20-23]. The average velocity of the elementary layers of the liquid relative to the nanoparticle is very small. The surface force field around the nanoparticle forms PMBLLM (LBL) with excess surface energy, and as the distance from the nanoparticle surface increases, the velocity vectors increase in a parabola. In fact, around a turbulently moving nanoparticle, a arises of "adhered" PMBLLM (LBL) (Figure 2), and which may not leave their host (nanoparticle) for a relatively long time. Moreover, the average thickness of this layer $\delta$ may be 1-2 orders of magnitude larger than the size of the nanoparticle itself. And this layer ends where the gradient of surface forces no longer acts. We will consider the relative microslips of the elementary layers of the liquid in PMBLLM as the mutual.

$\mathrm{L}$ - motion in the LBL.

Suppose that the forces act on the elementary volume of the liquid in the LBL:

1. The force of surface tension of a liquid:

$$
F_{\sigma}=\pi(d x) \cdot \sigma \cdot \cos \theta
$$

2. The force of gravity:

$$
m g=\rho \cdot \mathrm{g}(d \mathrm{x} d \mathrm{y} d \mathrm{z})
$$

3. Friction force:

$$
T=\mu \frac{d^{2} V_{z}}{d x^{2}}(d x d y d z)
$$

4. Inertia force:

$$
F_{i}=m b=\rho \cdot \frac{d V_{z}}{d \tau}(d x d y d z)
$$

5. Pressure force:

$$
P=-\frac{d p}{d z}(d x d y d z)
$$

The algebraic sum of all forces acting on the elementary volume of fluid in a LBL is zero.

Reducing by $(d x d y d z)$, we obtain the relation (21):

$$
\begin{aligned}
& \pi \cdot(d x) \cdot \sigma \cdot \cos \theta /(d x d y d z)+ \\
& \rho \cdot g-\frac{d P}{d z}+\mu \frac{d^{2} V_{z}}{d x^{2}}=\frac{\rho \cdot d V_{z}}{d \tau}
\end{aligned}
$$

We denote the differentiation and direction symbols in this differential equation, and the linear parameters of the elementary volume $d x, d y, d z$ are replaced by $l . \frac{d V}{d l} \approx \frac{V}{l} ; \frac{d V}{d \tau} \approx \frac{V}{\tau}$; By dividing all the terms of Eq. (21) by the magnitude $\frac{\rho V}{\tau}$, we obtain corresponding similarity numbers in the LBL (22-25).

At the same time, we substitute in these similarity numbers the approximate, averaged numerical values for the water under normal conditions. In this case, the remaining values are selected as:

- the average diameter of nanoparticle $\left(d \approx 20.10^{-9} \mathrm{~m}\right)$;

- a density of nanoparticle $\left(\rho_{n p}=2100 \mathrm{~kg} \cdot \mathrm{m}^{-3}\right)$;

- a density of the water $\left(\rho=1000 \mathrm{~kg} \cdot \mathrm{m}^{-3}\right)$;

- coefficient of surface tension of water $(\sigma=0.0725 \mathrm{~N} / \mathrm{m})$;

- average thickness of LBL $\left(\delta \approx 0.4 .10^{-6} \mathrm{~m}[15,16]\right)$;

- the hydrophilicity of the nanoparticle surface $(\cos \theta \approx 0.8)$.

- $\operatorname{Re} \approx 100000$, which corresponds to the developed $\mathrm{T}$ regime of the coolant flow.

The speed of motion of water layers in the LBL is about an order of magnitude less than the linear speed of motion. We are also guided by the formula for uniformly accelerated motion of a nanoparticle,

$$
l=V_{z} \cdot \tau ; V_{z} \approx \frac{V}{N} \approx \frac{V}{10} \approx \frac{0.1}{10} \approx 0.01 \mathrm{~m} \cdot \mathrm{s}^{-1}
$$

$\Delta p$ - the pressure difference in front of and behind the particle, $\mathrm{Pa} ; N=\frac{\rho \cdot V_{Z} \cdot l}{\mu} \approx 10.47-11.5$ [24].

1. Euler number $(\mathrm{Eu})$ in LBL:

$$
\begin{aligned}
& E u=\frac{P \cdot \tau}{\rho \cdot V \cdot l}=\frac{\Delta p}{\rho \cdot V_{z}^{2}}=\frac{\frac{\pi \mathrm{d}^{3} / 6 \cdot \rho_{n f}}{\pi \mathrm{d}^{2} / 2} \cdot \operatorname{Re}}{\rho \cdot V_{z}^{2}} \\
& =\frac{20 \cdot 10^{-9} \cdot 2100 \cdot 100000}{3 \cdot 1000 \cdot 0.01^{2}} \approx 14 ;
\end{aligned}
$$

In the Euler number (22), the pressure difference $\Delta p$ in front of the nanoparticle and behind it [20-23] is created by the inertial force. Instead of the difference in pressure, we substitute the force of gravity that acts on the hemisphere area in front of the nanoparticle. However, the turbulence of the coolant flow (and we determined this at the beginning of the modeling, since the heat exchangers operate in a turbulent mode) is not created due to the movement of the nanoparticle under the action of gravity, but due to the movement of the coolant at $\mathrm{Re} \geq 20000-100000$ or more. Therefore, in Euler's number, we multiply by $\mathrm{Re} \approx 100000$. We can assume that the turbulization of the flow is created due to centrifugal forces, 
then we multiply by the centrifugal factor. $\left(K_{C}=\omega^{2} R / g\right)$.

2. Surface number $(\mathrm{Su})$ in LBL:

$$
\begin{aligned}
& \text { Su }=\frac{1}{N} \cdot \frac{2 \cdot \pi \cdot \sigma \cdot \cos \theta}{\mu \cdot V_{z}}= \\
& \frac{1}{10} \cdot \frac{2 \cdot 3.14 \cdot 72.5 \cdot 10^{-3} \cdot 0.8}{1 \cdot 10^{-3} \cdot 0.01} \approx 3642 ;
\end{aligned}
$$

3. Reynolds number in (Re) in LBL:

$$
\begin{aligned}
& \frac{1}{\operatorname{Re}}=\frac{\mu \cdot V \cdot \tau}{l^{2} \cdot \rho \cdot V}=\frac{\mu}{\delta \cdot \rho \cdot V_{z}}= \\
& \frac{1 \cdot 10^{-3}}{0.4 \cdot 10^{-6} \cdot 1000 \cdot 0.01} \approx 250 ; \operatorname{Re} \approx 0.004
\end{aligned}
$$

4. Froude number (Fr) in LBL:

$$
F r=\frac{\rho \cdot g \cdot \tau}{\rho \cdot V}=\frac{g \cdot \tau \cdot \delta}{V \cdot l}=\frac{g \cdot \delta}{V_{z}^{2}}=\frac{9.8 \cdot 0.4 \cdot 10^{-6}}{0.01^{2}} \approx 0.039
$$

Based on such a comparative analysis, we see that the numbers responsible for the forces of friction and gravity in the LBL are very small compared to the forces of surface tension (the surface criterium is several orders of magnitude larger than the others). Consequently, all forces that act on a nanoparticle, including the Archimedes force, can be neglected, except for surface and inertia forces, which create rarefaction behind the direction of motion of the particle at a certain point in time and excess pressure in front of it (Figure 2 ). This fact is successfully proved by the authors using methods molecular dynamics [20-23]. In fact, the force of inertia is included in the Euler number in the LBL.

Let us consider the forces of pressure and the forces of surface tension in the LBL, acting on the elementary ring of the liquid (Figure 2).

1. The area of the elementary ring in LBL:

$$
d S=2 \pi \cdot r \cdot d r
$$

2. Pressure force acting on the elementary ring in LBL:

$$
d P=p \cdot 2 \pi \cdot r \cdot d r
$$

3. The force of surface tension of a liquid acting in the LBL:

$$
d F_{\tau}=2 \pi \cdot \sigma \cdot \cos \theta \cdot d r
$$

Let us equate the pressure forces (27) and surface forces (28) in LBL and integrate the equation, removing the constant values outside the integration sign, and also establish the integration limits. After integration, we obtain the relation (Figure 1) (29):

$$
\begin{gathered}
d P=\Delta p 2 \pi \int_{0}^{\delta} r d r=\Delta p \pi \delta^{2} \\
d F_{\Pi}=2 \pi \sigma \cos \theta \int_{R_{0}}^{R} d r \\
\Delta p \pi \delta^{2}=\pi \sigma \cos \theta d
\end{gathered}
$$

The difference in pressure $\Delta p$ in front and behind the nanoparticle is created by the inertial force, which is $m b$. Instead of the difference in pressure, we substitute the force of inertia that acts on the hemisphere area in front of the nanoparticle (30):

$$
\frac{\pi \mathrm{d}^{3} / 6 \cdot \rho_{n f} \cdot b \cdot \operatorname{Re}}{\pi \mathrm{d}^{2} / 2} \cdot \pi \cdot \delta^{2}=\pi \cdot \sigma \cos \theta \cdot d
$$

By shortening the values of $\mathrm{d}$ and $\pi$, we obtain the mean thickness of the LBL (31):

$$
\delta=1,732 \sqrt{\frac{\sigma \cdot \cos \theta}{b \cdot \rho_{n f} \cdot \operatorname{Re}}}
$$

Analyzing the numbers in LBL (22-25) and formula (31) obtained by us and returning to works [20-23], the qualitative following correlations can be observed:

- surface forces prevail in the LBL around a turbulently moving nanoparticle, and they are several orders of magnitude greater than all others;

- it is clearly seen from (Figure 1) [18] that the tendency to an increase in the coefficient of thermal conductivity correlates with an increase in the average diameter of nanoparticles, and this increase is greater for ethylene glycol solutions than for water. The specific surface energy of glycols fluctuates around $50.10^{-3} \mathrm{~J} / \mathrm{m}^{2}$, and of water - about $70.10^{-3}$ $\mathrm{J} / \mathrm{m}^{2}$. Based on formula (31) about $(20-30) \%$ increase for ethylene glycols in comparison with water is created due to the lower average value of the specific surface energy of the latters;

- with an increase in the diameter of nanoparticles and, accordingly, their weight in a liquid medium, the acceleration of the inertial forces of their chaotic motion increases, and the average thickness of the LBL decreases (31) (Figure 2), which contributes to an increase in heat transfer from nanoparticles to the liquid medium, because $\mathrm{L}$ - flow transfers the amount of heat much worse than $\mathrm{T}$ - flow;

- the viscosity of nanofluids decreases due to the fact that nanoparticles with a smaller average thickness of the LBL are less prone to conglomeration. In addition, the addition of various surfactants to nanofluids reduces the surface tension coefficient and also contributes to a decrease in the average thickness of the LBL. Formula (31) also confirms the experiments performed in the study [33] that the higher density of nanoparticles $\left(\mathrm{CuO}-6510 \mathrm{~kg} \cdot \mathrm{m}^{-3}\right)$ than $(\mathrm{ZnO}-5606$ $\mathrm{kg} . \mathrm{m}^{-3}$ ) contributes to a decrease in the average thickness of the LBL and this has a positive effect on the total thermal conductivity of the nanofluid.

In natural emulsion milk, the average diameter of fat globules ranges from about $d \approx(2.5-5) \cdot 10^{-6} \mathrm{~m}$. The specific surface energy of milk fat is about $35.10^{-3} \mathrm{~J} / \mathrm{m}^{2}$, and that of milk plasma is about $50.10^{-3} \mathrm{~J} / \mathrm{m}^{2}$. In this case, the gradient of the specific surface energy scalar is about 1.4 times. In the case of a metal nanoparticle in a liquid, the opposite is true. Its specific surface energy is about $(0.5-3) \mathrm{J} / \mathrm{m}^{2}$ [29-31, 32] and the specific surface energy of liquids is much less - about (20$70) .10^{-3} \mathrm{~J} / \mathrm{m}^{2}$. In this case, the specific surface energy scalar gradient is, on average, about 40 times. If the surface plasma energy in milk suppresses the specific surface energy of fat and forms balls with an average diameter of about $d \approx 3.10^{-6}$ $\mathrm{m}$, then the surface of a metal nanoparticle should create a field of action of surface forces approximately at a distance of $\delta \approx$ $100.10^{-9} \mathrm{~m}$. This gradient of the field of surface forces in the 
T- mode of motion of the nanoparticle creates a LBL, which is proportional to $\delta \approx 100.10^{-9} \mathrm{~m}$, with an average nanoparticle diameter of about $d \approx 20.10^{-9} \mathrm{~m}$. That is, the average length of the field of action of the surface forces is about (5-10) times greater than the average diameter of a nanoparticle.

It should be emphasized that in many heat exchange systems, LBL resistances are considered, which are even given a predominant role in the formation of the total thermal conductivity. However, based on our studies, in heat exchangers the resistivity of the LBL in the near-wall regions is tens of times higher, but their total thermal resistance is insignificant (about $1.5 \%$ ) due to the very small average thickness of the LBL [24]. In the case of nanoparticles, as we can see, the opposite is true average nanoparticle diameter about $d \approx 20.10^{-9} \mathrm{~m}$, and the average LBL minimal thickness is about $\delta \approx 100.10^{-9} \mathrm{~m}$. It is difficult to understand that in such systems, the thermals resistances of the LBLs is not considered. This reminds us of a picture of a floating fish in an aquarium, when we pay close attention to it, but we do not notice the aquarium. It is precisely properties of LBL, in particular its average thickness, that play a key role in changing the thermal conductivity and viscosity of nanofluids. It should be assumed that a randomly moving metallic nanoparticle facilitates the transfer of heat to the base fluid, and LBL around it creates additional thermal resistance. The change the average thickness of LBL by the change the average diameter of nanoparticles, the change their density and specific surface energy respectively, can control the heat transfer coefficients of nanofluids, that is, go to the rank of thermal nanotechnology.

We believe that this particular model also explains the fact that the addition of more than 5\% nanoparticles to the base fluid turns out to be ineffective, since all places for free turbulent motion are occupied due to a relatively very large average thickness LBL.

However, the most significant, in our opinion, inaccuracy in studying the behavior of nanofluids is that this system is considered in static, stationary conditions using the "classical", molecular viscosity and thermal conductivity coefficients. In addition, the surface forces are not taken into account, which are absolutely dominant in the LBL, which we proved above.

\subsection{Numerical equation for calculating the coefficients of convective (turbulent) thermal conductivity}

We replace the convection coefficient $h\left[\mathrm{~W} \cdot \mathrm{m}^{-2} \cdot \mathrm{K}^{-1}\right]$ in the Nusselt number (Eqns. 2, 3) with the convective (turbulent) thermal conductivity coefficient $k_{t u r b}$. $\left[\mathrm{W} \cdot \mathrm{m}^{-1} \cdot \mathrm{K}^{-1}\right]$. According to the theory of $\mathrm{J}$. Boussinesq, we introduce the concept of turbulent viscosity $\mu_{\text {turb }}$. $\left[\mathrm{kg} . \mathrm{s}^{-1} \cdot \mathrm{m}^{-1}\right]$ (32) of a fluid heat carrier, since a turbulent fluid is considered as Newtonian. The derivation and detailed analysis of Eq. (32) by the dimensional method is presented in our previous work [26].

$$
\mu_{\text {turb. }}=\left\langle\begin{array}{l}
W^{X} \cdot \sigma^{1} \cdot C_{P}^{(-0.5-0.5 X)} \\
\cdot R^{(-2 X)} \cdot K^{(-0.5-0.5 X)}
\end{array}\right\rangle: \frac{\sigma \cdot \cos \theta}{\sqrt{C_{P} \cdot 1^{0} K}}
$$

It also seems to us that the parameter of the linear velocity of the movement of the coolant $V\left[\mathrm{~m}_{\mathrm{s}} \mathrm{s}^{-1}\right]$, as well as mass velocity $V . \rho\left[\mathrm{kg} . \mathrm{m}^{-2} \mathrm{~s}^{-1}\right]$ in the Eq. (2) not fully reflect the flow characteristic. We propose to introduce the parameter of the volumetric flow rate of the coolant $W\left[\mathrm{~m}^{3} . \mathrm{s}^{-1}\right]$ in the Eq. (32) which characterizes the linear velocity and the parameter of the "free area" of the flow. And most importantly, we propose to introduce into the calculation the surface parameter responsible for the surface tension forces - the surface tension coefficient of the fluid $\sigma\left[\mathrm{N} . \mathrm{m}^{-1}\right]$.

The coefficient of convective (turbulent) thermal conductivity $k_{\text {turb }}$. $\left[\mathrm{W} \cdot \mathrm{m}^{-1} \cdot \mathrm{K}^{-1}\right.$ ] with turbulent movement of a liquid or gas heat carrier will depend on the following main factors:

- turbulent dynamic viscosity of the liquid

- volumetric flow rate of the heat carrier

$\mu_{\text {turb. }}$ [Pa. s];

- coefficient of surface tension of the liquid $W\left[\mathrm{~m}^{3} \cdot \mathrm{s}^{-1}\right]$

- specific heat capacity of liquid heat carrie $\sigma\left[\mathrm{N} \cdot \mathrm{m}^{-1}\right]$

- linear dimension, e.g. pipe radius $C_{P}\left[\mathrm{~J}^{\mathrm{kg}} \mathrm{kg}^{-1} \mathrm{~K}^{-1}\right]$ $R[\mathrm{~m}]$.

As a result of comparing the turbulent and molecular viscosity or turbulent and molecular thermal conductivity of the heat carrier fluid, we obtain the numerical Eq. (33) $[25,26]$

$$
\begin{aligned}
& \frac{k_{\text {turb. }}}{C_{P} \cdot \mu}=\frac{\sigma \cdot \cos \theta}{\mu \cdot \sqrt{C_{P} \cdot 1^{0} K}}\left(\frac{\sqrt{C_{P} \cdot 1^{0} K}}{V}\right)^{-X} ; \\
& \text { or } \quad \frac{\mu_{\text {turb. }}}{\mu}=B l_{\text {turb. }} / B l ;
\end{aligned}
$$

It has only one unknown exponent $(-X)$, which shows the degree of turbulence of the liquid flow of the heat carrier. $B l_{\text {turb. }}$. number is responsible for the convective component of the flow, and the $B l$ number - for the molecular one. Turbulent and molecular viscosities depend significantly on temperature. The mutual dependence of these quantities has been studied rather thoroughly and shown in the work [26].

Since in section 3.1 of this work, comparing the similarity numbers in the laminar boundary layer (LBL) at the interface between a solid nanoparticle and a liquid coolant, we proved that surface forces dominate by several orders of magnitude over all other forces, the obtained similarity numbers $B l$ and $B l_{\text {turb. }}[25,26]$ have surface characteristics - the surface coefficient tension and hydrophilicity of the wetting surface, which is not observed in the classical ones.

\subsection{Analysis of the molecular $B l$ number according to the law of the distribution of temperatures and velosities fields}

We suggest taking a close look at formula (34), i.e. think about the physical meaning of the $B l$ number $[25,26]$. Using the theory of similarity, we can write the following conclusions:

Molecular $B l$ number, that we deduced in the works by the dimensional analysis method is the ratio products of internal friction forces and cohesion forces to surface tension forces in a liquid coolant, i.e. (34):

$$
\begin{aligned}
& B l=\frac{\mu \cdot \sqrt{C_{p} \cdot 1^{0} K}}{\sigma \cdot \cos \theta} \\
& =\frac{\text { Friction internal force } \cdot \text { Cohesion force }}{\text { Interfacial Surface tension force }}
\end{aligned}
$$

If we look differently at the molecular number $B l$ (see [25, $26]$ ), then this is the ratio of molecular viscosity to transitional viscosity in LBL (35) and, at the same time, is the ratio of the average velocity of thermal movement of molecules of fluid to the average relaxation rate of these molecules, i.e. (36): 


$$
B l=\frac{\mu}{\frac{\sigma \cdot \cos \theta}{\sqrt{C_{p} \cdot 1^{0} K}}}
$$$$
=\frac{\text { molecular } \cdot \text { viscosity in } \mathrm{LBL}}{\text { transitional } \cdot \text { viscosity in LBL }}=\frac{P a \cdot s}{P a \cdot s}
$$

$$
B l=\frac{\sqrt{C_{p} \cdot 1^{0} K}}{\frac{\sigma \cdot \cos \theta}{\mu}}=
$$

$$
\frac{\text { the thermal rate of the molecules }}{\text { the relaxation rate of the molecules }}=\frac{\mathrm{m} \cdot \mathrm{s}^{-1}}{\mathrm{~m} \cdot \mathrm{s}^{-1}}
$$

If we multiply the numerator and denominators of formula (36) by the specific heat of the heat transfer fluid, we get the ratio of the thermal conductivities in LBL, that is, the flow characteristics in LBL that transfer the amount of heat through LBL, which means that they are responsible for raising or lowering the temperature in individual elementary layers of the LBL.

If the number $B l(34)$ is represented in the form (35), it is similar to the left side of the Eq. (1), and if it is represented in the form (36), then it is similar to the right side of equation (1). The number $B l$ contains the distribution law (1) i.e., the simultaneous distribution of the temperatures and velocities fields in elementary layers of a liquid at the molecular level. It is noteworthy that at a temperature of about $20^{\circ} \mathrm{C}$ for water, the molecular number $B l$ is approximately equal to 1 (see [25]), which indicates the balance of all these forces in so-called normal terrestrial.

\subsection{Analysis of the turbulent number $B l_{\text {turb. according to }}$ the law of the distribution of temperatures and velosities fields}

The dimensionless number $B l_{\text {turb. }}$ is (37) [26]. When we consider the turbulent part of the flow of the heat carrier, the linear velocity makes the prevailing value in the formation of its structure. The speed is greater - the turbulization is more intense.

$$
\begin{aligned}
& B l_{\text {turb. }}=\frac{\mu_{\text {turb. }} \cdot \sqrt{C_{p} \cdot 1^{0} K}}{\sigma \cdot \cos \theta} \\
& =\frac{\text { Friction force in turbulent flow } \cdot \text { Cohesion force }}{\text { Interfacial Surface tension force }}
\end{aligned}
$$

We perceive the turbulent flow zone according to the model of J. Boussinesq. Moreover, we see that the turbulent number $B l_{\text {turb. }}$. differs from the molecular one only in the value of turbulent viscosity. Absolutely clearly, this coincides with the conclusion of Italian scientists that the laminar zone of the flow (LBL) completely controls its turbulent zone [34].

In particular, we presented the final formula for calculating the turbulent number $B l_{\text {turb }}$, which has the form (38) [26].

$$
\begin{gathered}
B l_{\text {turb }}=\left(\frac{\sqrt{C_{P} \cdot 1^{0} K}}{V}\right)^{-X} \text { or } \\
B l_{\text {turb } b}=\frac{1}{B l}\left(\frac{\sqrt{C_{p} \cdot 1^{0} K}}{V}\right)^{-X} \frac{\mu \sqrt{C_{p} \cdot 1^{0} K}}{\sigma \cdot \cos \theta} .
\end{gathered}
$$

Similarly to the molecular number, if we multiply the numerator and denominator of the left side of Eq. (38) by the specific heat, we obtain of attitude the maximal turbulent thermal conductivity in the central part of the coolant flow to is minimal value (transitional value) at the end of LBL (see works $[25,26]$. Turbulent thermal conductivity obviously corresponds to the transfer of a large amount of heat and temperature, and therefore the relation on the left side of formula (38) is similar to the relation on the left side of Eq. (1). On the right side of relation (38) we have the ratio of the value reflecting the average thermal velocity of the molecules of the liquid medium (maximum) to the linear velocity of the coolant flow (minimum). From the above analysis it can be seen that the turbulent number $B l_{\text {turb. }}$. also contains distribution law, which is contained in formula (1).

\subsection{Simulation of a force field acting on a nanoparticle based on hydrodynamic similarity taking into account MDM}

In the case of turbulent (chaotic) movement of the coolant, nanoparticles also move chaotically, being covered by LBLs with laminar and transient modes (Figure 2). The interfacial surface tension force and the Stokes force can randomly change their vector and scalar, since the nanoparticle collides with the molecules of the base fluid under conditions that are almost equivalent in momentum [20-22]. Based on the main conclusions [23] based on MDM, the interfacial surface tension force under unsteady conditions can be $(2-3)$ times greater than the Stokes drag force.

However, under stationary conditions, these forces are approximately equal [23]. Since we have a turbulent fluid, we introduce the value of the turbulent viscosity into the classical Stokes formula, following the Boussinesq principle. We also take into account the hydrophilicity of the wetted surface of the nanoparticle, which changes depending on the contact angle at a certain point in time. Based on the above analysis, we write down Eq. (39):

$$
\pi d \sigma \cos \theta=(2 \div 3) \cdot 3 \cdot \pi d V \mu_{t u r b}
$$

It is noteworthy that this simulation of the behavior of nanoparticles in a turbulent flow at the nanoscale completely coincides with the simulation of the behavior of dispersed liquid droplets at the macroscale, which is confirmed experimentally [35].

Substituting the value of the turbulent viscosity from the turbulent number $B l_{\text {turb }}$, taking into account the ratio (39), we obtain the ratio (40):

$$
\left(\frac{\sqrt{C_{p} \cdot 1^{0} K}}{(3-9) \cdot V}\right)=\left(\frac{\sqrt{C_{p} \cdot 1^{0} K}}{V}\right)^{-X} 1 / B l ;
$$

Solving Eq. (40) with respect to $(-X)$, we obtain a complex of formulas (41) (Table 3). Based on the fact that the interfacial tension force at the interface between the nanoparticle surface and the coolant is not stationary, it depends on the contact angle and at first the motion can be 2-3 times greater than the Stokes resistance force, and only in the steady state [23] asymptotically approaches it, equations (Table 3 ) do not have a unique universal solution. Through painstaking computer calculations, we averaged the $(-X)$ values, and obtained ranges for using some equations (Table 3 ). In this case, the number under the logarithm can change namely: 
Table 3. Determination of the indicator $(-X)$ (Formula 41)

\begin{tabular}{|c|c|c|}
\hline$B l$ & Dynamic viscosity & Degree (-X)(Formula 41) \\
\hline$B l \geq 1$ & $\begin{array}{l}2 \mu_{\mathrm{H}_{2} \mathrm{O}}\left(20^{0} \mathrm{C}\right) \geq \mu \geq 1.5 \cdot \mu_{\mathrm{H}_{2} 0}\left(20^{\circ} \mathrm{C}\right) \\
\text { milk, oils, aqueous solutions of glycols }\end{array}$ & $-X=1-\frac{\ln (1 / B l)+\ln 6}{\ln \frac{\sqrt{C_{p} \cdot 1^{0} K}}{V}}$ \\
\hline$B l \leq 1$ & $\begin{array}{l}\quad \mu \leq \mu_{H_{2} \mathrm{O}}\left(20^{\circ} \mathrm{C}\right) \\
\text { water at high temperatures }\end{array}$ & $-X=1-\frac{\ln (1 / B l)+\ln 3}{\ln \frac{\sqrt{C_{p} \cdot 1^{0} K}}{V}}$ \\
\hline$B l \geq 1$ & $\begin{array}{l}1.5 \mu_{\mathrm{H}_{2} \mathrm{O}}\left(20^{0} \mathrm{C}\right) \geq \mu \geq \mu_{\mathrm{H}_{2} \mathrm{O}}\left(20^{0}\right) \\
\text { milk, glycols at high temperatures }\end{array}$ & $-X=1-\frac{\ln (1 / B l)+\ln 4}{\ln \frac{\sqrt{C_{p} \cdot 1^{0} K}}{V}}$ \\
\hline$B l \geq 1$ & $\begin{array}{l}\qquad \mu \geq 2 \cdot \mu_{H_{2} 0}\left(20^{\circ} \mathrm{C}\right) \\
\text { glycols at low temperatures, cream, butter, cooking, sugar and salt solutions }\end{array}$ & $-X=1-\frac{\ln (1 / B l)+\ln 5}{\ln \frac{\sqrt{C_{p} \cdot 1^{0} K}}{V}}$ \\
\hline$B l \leq 1$ & $\begin{array}{c}\mu \leq \mu_{\mathrm{H}_{2} \mathrm{O}}\left(20{ }^{0} \mathrm{C}\right) \\
\text { solutions of alcohols, gasolines, kerosene }\end{array}$ & $-X=1-\frac{\ln (1 / B l)+\ln 9}{\ln \frac{\sqrt{C_{p} \cdot 1^{0} K}}{V}}$ \\
\hline
\end{tabular}

In view of the fact that the use of formulas is some inconvenience, we propose a slightly different, semi-empirical and semi-analytical approach to this problem.

As a comparison, we also present formula (42) for calculating the turbulent viscosity of air flows [25, 26].

$$
\begin{gathered}
\mu_{\text {turb. }}=\frac{\mu \cdot a \sqrt{2 \mathrm{Re}}}{0.769} \\
a=(0.05-0.08)[25] ;
\end{gathered}
$$

Based on the foregoing, we propose to equate formulas (42 and 33) with each other in order to be able to obtain the value of degree $(-X)$ in a more convenient way and to obtain the relation (43):

$$
-X=\frac{\ln \frac{a \cdot \sqrt{2 \mathrm{Re}}}{0.769 \cdot 1 / B l}}{\ln \frac{\sqrt{C_{p} \cdot 1^{0} K}}{V}}
$$

\subsection{Classical and new express-method for evaluating the efficiency of using nanofluids in various heat exchangers}

To date, we have performed numerous calculations for shell and tube and plate heat exchangers using the classical and our new method, which show that the new method is more accurate and correct when using various mixtures of nanofluids. The data of these calculations are extensive, and their comparative results cannot be physically presented in this work. However, in order to confirm the correctness of our proposed method, we present a well-known rapid test of the effectiveness of the use of specific nanofluids in heat exchange equipment, the main idea of which is as follows [17]:

- in a turbulent mode of flow of coolants in heat exchange equipment and at a fixed flow rate, when the calculations are carried out according to the classical scheme, that is, using the classical Nusselt, Reynolds and Prandl numbers and classical equations such as (3), the effective action of the nanofluid is observed with a positive ratio of its thermal conductivity and viscosity [17];

- the change in the convection coefficient of the liquid heat carrier $\mathrm{h}$ upon addition of the corresponding nanoparticles to it is proportional to the change in the corresponding complex [17], which is easily derived from equations of the type (3), for example, for the shell of a shell-and-tube heat exchanger, where water is supplied as model nanofluids of the heat carrier with the addition of the corresponding ethylene glycols:

$$
\begin{gathered}
N u_{h}=0.24 \cdot \operatorname{Re}_{h}^{0.6} \operatorname{Pr}_{h}^{0.36} ; \\
\frac{h_{h} \cdot D}{k}=0.24 \cdot\left(\frac{V \cdot D \cdot \rho}{\mu}\right)^{0.6}\left(\frac{C_{P} \cdot \mu}{k}\right)^{0.36} \text { i.e. } \\
h_{h} \sim \frac{k^{(1-0.36)}}{\mu^{(0.6-0.36)}}=\frac{k^{0.64}}{\mu^{0.24}} ;
\end{gathered}
$$

- numerical equation when using nanofluid such as $\mathrm{TiO}_{2}$, e.g., $N u=0.021 \operatorname{Re}^{0.8} \mathrm{Pr}^{0.5}$ [4], (analogous equation without using nanofluid $N u=0.021 R^{0.8} \mathrm{Pr}^{0.43}$ );

- let us increase the exponent in the Eq. (3) for the shell-andtube heat exchanger shell by 0.07 , respectively, eq.:

$$
\begin{aligned}
& N u_{h}=0.24 \cdot \operatorname{Re}_{\mathrm{h}}^{0.6} \operatorname{Pr}_{h}^{(0.36+0.07)=0.43} ; \\
& \frac{h_{h} \cdot D_{h}}{k_{h}}=0.24 \cdot\left(\frac{V_{h} \cdot D_{h} \cdot \rho_{h}}{\mu_{h}}\right)^{0.6}\left(\frac{C_{P . h} \cdot \mu_{h}}{k_{h}}\right)^{0.43}
\end{aligned}
$$

- complex when using nanofluid for the shell-and-tube heat exchanger shell (44b):

$$
h_{h} \sim \frac{k_{h}{ }^{(1-0.43)}}{\mu_{h}^{(0.6-0.43)}} \sim \frac{k_{h}^{0.57}}{\mu_{h}^{0.17}} ;
$$

- if the complex $h_{h} \sim \frac{k^{0.64}}{\mu^{0.24}}$ a result of additions to the coolant of the corresponding nanoparticles, it grows, then we observe a positive trend in the improvement of heat transfer, when it decreases - negative [17]. 
For the classic express method (classic numerical equation for shell-and-tube heat exchanger tubes (3):

$$
\begin{gathered}
N u_{c}=0.023 \cdot \operatorname{Re}_{c}{ }^{0.8} \operatorname{Pr}_{c}^{0.43} ; \\
\frac{h_{c} \cdot D_{c}}{k_{c}}=0.023 \cdot\left(\frac{V_{c} \cdot D_{c} \cdot \rho_{c}}{\mu_{c}}\right)^{0.8}\left(\frac{C_{P . c} \cdot \mu_{c}}{k_{c}}\right)^{0.43} \\
h_{(\text {milk }) c} \sim \frac{k_{c}^{(1-0.43)}}{\mu_{c}^{(0.8-0.43)}}=\frac{k_{c}^{0.57}}{\mu_{c}^{0.37}}
\end{gathered}
$$

\subsubsection{Classical express - method}

1. Therefore, the complexes for pure hot water, hot water with a mixture of $\mathrm{H}_{2} \mathrm{O}+\mathrm{EG}(60: 40)$ and hot water with a mixture of $\mathrm{H}_{2} \mathrm{O}+\mathrm{EG}(60: 40)+1.5 \% \mathrm{TiO}_{2}$ and is thermophysical characteristics at $70^{\circ} \mathrm{C}$ are presented in Table 4.

\begin{tabular}{|c|c|c|c|}
\hline Volume & $\mathrm{H}_{2} \mathrm{O}$ & $\begin{array}{c}\mathrm{H}_{2} \mathrm{O}+\mathrm{EG} \\
(60: 40)\end{array}$ & $\begin{array}{c}\mathrm{H}_{2} \mathrm{O}+\mathrm{EG} \\
(60: 40)+ \\
1.5 \% \mathrm{TiO}_{2} \\
\end{array}$ \\
\hline Complexes $(44 a, 44 b)$ & 5.026 & 3.017 & 2.042 \\
\hline Thermal conductivity & 44.39 & 76.24 & 84.24 \\
\hline $\begin{array}{l}\text { turb. } \\
k_{\text {turb. } h}, \mathrm{~W} \cdot \mathrm{m}^{-1} \cdot \mathrm{K}^{-1}(46)\end{array}$ & $\rightarrow$ & $\rightarrow$ & $\rightarrow$ \\
\hline Density, $\rho_{h}, \mathrm{~kg} \cdot \mathrm{m}^{-3}$ & 970 & 1033 & 1081 \\
\hline $\begin{array}{c}\text { Dynamic viscosity. } 10^{3} \\
\mu_{h}, \mathrm{~kg} \cdot \mathrm{m}^{-1} \cdot \mathrm{s}^{-1}\end{array}$ & 0.41 & 1.11 & 1.48 \\
\hline $\begin{array}{l}\text { Surface tension. } 10^{3} \\
\sigma_{h}, \text { N.m }\end{array}$ & 62.25 & 51.19 & 51.00 \\
\hline $\begin{array}{l}\text { Heat capacity: } \\
C_{p h}, \mathrm{~J}_{\mathrm{kg}}^{-1} \cdot \mathrm{K}^{-1}\end{array}$ & 4198 & 3636 & 3463.3 \\
\hline $\begin{array}{c}\text { Thermal conductivity: } \\
k_{h}, \\
\text { W. } \mathrm{m}^{-1} \cdot \mathrm{K}^{-1}\end{array}$ & 0.670 & 0.438 & 0.501 \\
\hline $\cos \theta$ & 0.850 & 0.795 & 0.880 \\
\hline $\begin{array}{l}\text { The degree of flow } \\
\text { turbulization, }\left(-X_{h}\right)\end{array}$ & $\begin{array}{c}\mathbf{0 . 6 0 3} \\
\rightarrow\end{array}$ & $\begin{array}{c}0.823 \\
\rightarrow\end{array}$ & $\begin{array}{c}0.834 \\
\rightarrow\end{array}$ \\
\hline
\end{tabular}

Table 4. Complexes and thermophysical characteristics of hot water and corresponding mixtures

(arrows indicate the direction of increasing value)

2. Of course, we carried out full calculations of the shelland-tube heat exchanger and obtained the following data:

- the convection coefficient of the $\mathrm{H}_{2} \mathrm{O}+\mathrm{EG}$ mix (60:40) in comparison with pure water at a temperature of $70^{\circ} \mathrm{C}$ decreased by $(-4.5) \%$, and with the use of $\mathrm{TiO}_{2}$ nanoparticles by $(-1.5) \%$.

3. The calculation of the complex in paragraph 1 showed that the use of classical numerical equations for calculating heat exchangers using nanofluids at high temperatures $\left(70^{\circ} \mathrm{C}\right)$ is not accurate and inherently erroneous. Complex $(44 \mathrm{a}, \mathrm{b})$ in Table 4 decreases, which should indicate a decrease in the heat transfer coefficient when using $\mathrm{H}_{2} \mathrm{O}+\mathrm{EG}(60: 40)+1.5 \% \mathrm{TiO}_{2}$, but the results [36] and our computer analysis [16], indicate an increase.

It should be noted that so far we have focused on the water heat carrier and considered the possibility of intensifying heat transfer by introducing various additives into the water in the form of ethylene and propylene glycols, nanoparticles, etc. But in addition to water, the possibility of adding SAS to liquid food products, for example, milk, was considered. Formula (31) clearly shows that the introduction of a small amount of SAS into a liquid food product increases its turbulent thermal conductivity, since they, as a rule, significantly reduce the surface tension coefficient of the coolant. Our previous work [37] is devoted to these and other issues, where a wide range of natural vegetable oils in amounts up to $1 \%$ or less was considered as additives to milk. In relation to milk, these vegetable oils are SAS, and their introduction in small quantities helps to increase the nutritional value of milk, since they are source of unsaturated fatty acids and other valuable trace elements that are not synthesized in the human body.

Let us conduct an appropriate express assessment of the effectiveness of pumpkin seed oil use, as the most suitable for milk in terms of SAS and trace element composition [37]. The thermal conductivity of pumpkin seed oil is 3.9 times lower than that of milk $\left(k_{\mathrm{oil}}=0.146 ; k_{\text {milk(c) }}=0.5698\right)$ [38], the addition of $+0.5 \%$ pumpkin seed oil SAS reduces the thermal conductivity milk up to $k_{\text {milk(c) }}=0.5562 \mathrm{~W} \mathrm{~m}^{-1} . \mathrm{K}^{-1}$.

Therefore, the complexes and thermophysical characteristics of cold milk and corresponding mixture at $42.5^{\circ} \mathrm{C}$ are presented in Table 5.

\begin{tabular}{|c|c|c|}
\hline Volume & $\begin{array}{l}\text { Cold } \\
\text { milk }\end{array}$ & $\begin{array}{l}\text { Cold milk } \\
+0,5 \% \text {, } \\
\text { pumpkin } \\
\text { oil (SAS) }\end{array}$ \\
\hline \multirow{2}{*}{ Complexes (44a, 44b) } & 9.49 & 9.43 \\
\hline & $\leftarrow$ & $\leftarrow$ \\
\hline Thermal conductivity turb., $k_{\text {turb.c }}$, & 58.70 & 66.38 \\
\hline $\mathrm{W} \cdot \mathrm{m}^{-1} \cdot \mathrm{K}^{-1}(46)$ & $\rightarrow$ & $\rightarrow$ \\
\hline Density, $\rho_{c}, \mathrm{~kg} \cdot \mathrm{m}^{-3}$ & 1020 & 1020 \\
\hline Dynamic viscosity $\cdot 10^{3}: \mu_{c}, \mathrm{~kg} \cdot \mathrm{m}^{-1} \cdot \mathrm{s}^{-1}$ & 0.96 & 0.94 \\
\hline Surface tension: $\sigma_{c} \cdot 10^{-3}, \mathrm{~N}^{-1}$ & 47.75 & 35.25 \\
\hline Heat capacity: $c_{p c}, \mathrm{~J} \mathrm{~kg}^{-1} \cdot \mathrm{K}^{-1}$ & 3914 & 3914 \\
\hline Thermal conductivity: $k_{c}, \mathrm{~W} \cdot \mathrm{m}^{-1} \cdot \mathrm{K}^{-1}$; & 0.569 & 0.556 \\
\hline \multirow{2}{*}{ The degree of flow turb., $\left(-X_{c}\right)$} & 0.732 & 0.759 \\
\hline & $\rightarrow$ & $\rightarrow$ \\
\hline \multirow{2}{*}{$\begin{array}{l}\text { Average linear flow rate of the } \\
\text { coolant, } V_{c}, \mathrm{~m}_{\mathrm{s}}^{-1} \\
\cos \theta\end{array}$} & 0.659 & 0.659 \\
\hline & 0.70 & 0.95 \\
\hline
\end{tabular}

Table 5. Complexes and thermophysical characteristics of cold milk and corresponding mixture

The calculation shows that the complex does not increase, but slightly decreases due to the very low percentage of pumpkin oil SAS in the milk $(0.5 \%)$.

\subsubsection{New express - method}

All thermophysical characteristics are identical as for the classical calculation (Table 1) at a temperature of $70^{\circ} \mathrm{C}$ (the corresponding mixtures are similar to those used for calculations in [16]):

We remind you that in the paragraph 3.1 of this work, we replaced the heat transfer coefficient $\boldsymbol{h}$ by the turbulent thermal conductivity coefficient $\boldsymbol{k}_{\text {turb. }}$., Since they have almost the same physical meaning. In addition, in some works, for example [39], in there is a thesis that the convection coefficient does not fully reflect the turbulent and thermal characteristics of the heat carrier fluid.

1. From the power-law Eq. (33), the turbulent heat conductivity in the equation is equal to (46):

$$
k_{\text {turb. }}=(\sigma \cdot \cos \theta) \sqrt{C_{P} \cdot 1^{0} K}\left(\frac{\sqrt{C_{P} \cdot 1^{0} K}}{V}\right)^{-X}
$$




$$
k_{\text {turb. }}=k_{\text {trans. }}\left(\frac{\sqrt{C_{P} \cdot 1^{0} K}}{V}\right)^{-X}
$$

It should be understood that the factor $k_{\text {trans. }}=\left(\sigma_{h}\right.$. $\cos \theta) \sqrt{C_{P} \cdot 1^{0} K}$ in Eq. (46), which has the dimension of thermal conductivity $\left(\mathrm{W} \cdot \mathrm{m}^{-1} \cdot \mathrm{K}^{-1}\right)$, is the transitional thermal conductivity at the interface between the LBL and the turbulent flow, that is, the thermal conductivity in the transitional layer (see [26]). The dimensionless number $B l_{\text {turb. }}=\left(\sqrt{C_{P} \cdot 1^{0} K} / V\right)^{-X}$ is the ratio of the average speed of the thermal motion of the molecules of the liquid coolant to its linear speed in the exponent $(-X)(43)$. Where $(-X)$ is, the ratio of the coefficients of turbulent exchange of impulses $A \tau$ and heat $A q$ in formula (1) in the middle of the flow and is about $\approx 0.769$. Previous works $[25,26]$ were devoted to these questions.

2. Consequently, the values of turbulent thermal conductivities (46) for pure hot water, hot water with a mixture of $\mathrm{H}_{2} \mathrm{O}+\mathrm{EG}(60: 40)$ and hot water with a mixture of $\mathrm{H}_{2} \mathrm{O}+$ EG (60:40) $+1.5 \% \quad \mathrm{TiO}_{2}$ with the corresponding thermophysical characteristics are presented in Table 4.

Similar calculations using the formula (46) are made for a mixture of cold milk $+0.5 \%$, pumpkin oil (SAS) and are presented in Table 5 .

As can be seen from the calculations, the turbulent thermal conductivities of hot water is constantly increasing under the influence of the corresponding mixes. A similar situation is with cold milk, the turbulent thermal conductivity of which increases under the influence of $0.5 \%$ pumpkin oil.

This calculation, made on the basis of the new numerical Eq. (33) using formula (46), fully correlates with experiments [18], where an improvement in molecular thermal conductivity for solutions with ethylene glycol at a level of $+(30-40) \%$ is observed in comparison with pure water (Figure 1). The same calculations carried out using the classical Eq. (3) using the complex (44a-b) show the opposite - a decrease of - (4.5)\%. That is, we see that the classical equation is insensitive and does not "see" the presence of nanoparticles in nanofluids, where surface forces prevail. Because of this discrepancy, many researchers have resorted to various amendments to the classical equations [4-15], but again without taking into account the prevailing surface forces. Obtaining these equations requires extensive expensive experiments, they take on a complex, cumbersome form and become practically unsuitable for engineering express calculations of heat exchangers.

If we are guided by the concepts of turbulent viscosity and thermal conductivity and consider the $\mathrm{T}$ - flow taking into account surface forces, which is mandatory when using nanofluids, the positive effect is achieved primarily from a change in the degree of turbulence of the coolant $(-X)$, which in turn depends on the whole a number of factors included in the formula (43). It is interesting to understand that the turbulent thermal conductivity, according to formula (43), does not depend on the molecular viscosity of the coolant, as is proposed in most classical works related to nanofluids, but on the turbulent viscosity (43). Most studies assume that the addition of nanoparticles should reduce molecular viscosity, but this never happens. When nanoparticles are added, the molecular viscosity of the heat carrier, as a rule, increases (Table 4), and the turbulent thermal conductivity, primarily depends on the degree of flow turbulence $(-X)$, where a number of factors are present (43).

3. Of course, we carried out a complete calculation of the shell-and-tube heat exchanger using a new method and obtained the following data:

- the heat transfer coefficient of the mixture $\mathrm{H}_{2} 0+\mathrm{EG}(60: 40)$ in comparison with pure water at a temperature of $70^{\circ} \mathrm{C}$ increased by $(+12.86) \%$, and with the use of $\mathrm{TiO}_{2}$ nanoparticles by $(+14.45) \%$;

- if to the second heat carrier (milk) add mix ( $0.5 \%$ pumpkin oil), then the increase in the heat transfer coefficient is $(+16.75) \%$.

4.The calculation of the complex in point 3 , which increases with the use of mixtures, and the complete calculation of the shell-and-tube heat exchanger in point 4 showed that the use of the new numerical equation for the calculation of heat exchangers using nanofluids at high temperatures $\left(70^{\circ} \mathrm{C}\right)$ is accurate and reliable, which is confirmed by numerous experiments, for example, carried out in $[18,36]$ and our computer experiment [16]

5. We remind you that examples of complete calculations of a plate heat exchanger by the classical and new methods are given in our previous work [16]. However, in this work, we present comparative calculations using the mixtures $\mathrm{H}_{2} \mathrm{O}+\mathrm{EG}$ (60:40) and $\mathrm{H}_{2} \mathrm{O}+\mathrm{EG}(60: 40)+1.5 \% \mathrm{TiO}_{2}$, which fully confirm the reliability of using the new method and a new numerical equation when calculating heat exchange equipment when using nanofluids.

It should be emphasized that the exponents $(-X)$ calculated by us analytically in Tables 4,5 correlate well with the experimental and theoretical works presented in the monograph [2], where they were determined at the level of 0.769. However, in the indicated works, the surface characteristics of coolant flows do not appear, since at the time of writing these works, scientists were not engaged in nanofluids, but carried out their experiments, mainly on model fluids such as water. In this case, the classical numerical equations with the classical similarity numbers $\mathrm{Nu}, \mathrm{Re}, \mathrm{Pr}$ fully satisfied the accuracy of the calculations. In nanofluids at the nanoparticle - liquid flow boundary, as we proved in this work, surface forces prevail. Therefore, the classical equations fail and are improved by many scientists by adding various corrections for the concentration and fractional composition of nanofluids. The theoretical curves we obtained in [26] are almost analogous to the theoretical and experimental curves from the corresponding works [2] (see pages), which indicates the acceptability of our approach.

3.6.3 Evaluation of the effectiveness of the application of the classical and new numerical equations in the calculation of heat exchangers with nanofluidic coolants

Let's present the classical (2) and new (46) numerical equation in expanded form respectively:

$$
\begin{gathered}
\left(\frac{h \cdot D}{k}\right)^{1}=B \cdot\left(\frac{V \cdot D \cdot \rho}{\mu}\right)^{X}\left(\frac{C_{P \cdot} \cdot \mu}{k}\right)^{Y} ; \\
k_{\text {turb.h }}=\left(\sigma_{h} \cdot \cos \theta\right) \sqrt{C_{P} \cdot 1^{0} K}\left(\frac{\sqrt{C_{P} \cdot 1^{0} K}}{V}\right)^{-X}
\end{gathered}
$$

In the first classical numerical equation on the left is the Nusselt number, and on the right is the empirical constant $B$, 
as well as the Reynolds and Prandtl numbers with empirical indices $X$ and $Y$. The values of $B, X, Y$ analytically not interconnected in any way and do not depend on external factors (temperature coolant, the concentration of nanoparticles in it, etc.), but are detected by careful, expensive and laborious experiments, as evidenced by many works [415]. These equations sometimes take on a very complex and cumbersome form and become problematic for quick analytical express calculations of heat exchangers.

The second numerical equation, in our opinion, is an analytical analogue of Eq. (1), which was experimentally obtained by [1] and later duplicated by many authors, both analytically and experimentally on the principles of fluid mechanics [2] (see pages).

In fact, this is the ratio of turbulent and molecular thermal conductivity or viscosity of a structured flow of a liquid heat carrier and its comparison with the ratio of the average velocity of thermal motion of liquid molecules to the average linear velocity of the flow (see $[25,26]$ ).

However, the fundamental difference of this equation in the use of a surface parameter - the surface tension coefficient (or surface energy) liquid coolant, which becomes dominant under the conditions of using nanofluids, and turbulent characteristics flow - turbulent viscosity and thermal conductivity.

In deriving this equation, we took into account the following classical and modern studies:

- Eq. (33) is analytical using the new similarity numbers $B l$ and $B l_{\text {turb. }}$ obtained by us earlier $[25,26]$;

- the exponent $(-X)$ is actually an analytical expression of the turbulent Prandtl number, which obeys the logarithmic distribution law and correlates with his classical studies;

- when deriving the number $(-X)$ of formulas (41 or 43$)$, we used modern studies in the field of MDM from the corresponding works [20-23], where the relations of the Stokes resistance force and, as we have proved, the surface tension forces acting on a nanoparticle under non-stationary and stationary conditions of motion in a liquid medium. In this case, we used the classical Stokes formula, as well as the value of the turbulent viscosity of the coolant, than we used the analytical approaches of J. Boussinesq;

- in the analytic formula (43), we did not do without the Reynolds number, which made us closer to his classical studies;

- Eq. (33) in form is actually an analytical analogue of the power-law numerical empirical Nusselt equations;

- we have taken into account the modern research of Italian scientists Quadrio M., Ricco P. in the field of fluid mechanics that the laminar part of the fluid flow (LBL) completely controls its turbulent part [34]. This is evidenced by the similarity numbers themselves, obtained by us earlier $[25,26]$. Molecular number $B l$ simultaneously serves as a "constant" and a number that stands for the molecular component of the flow of a liquid or gaseous coolant sensitive to changes in external factors (temperature, concentration of nanoparticles, etc.) (Figure 3).

The $B l_{\text {turb. }}$ number is responsible for the turbulent component, which depends, first of all, on the ratio of the rate of thermal motion molecules to the linear the speed of movement $V$ of the coolant. The degree of flow turbulization $(-X)$, which is quite simply analytically found from our formulas (41 or 43).

Molecular and turbulent $B l$ and $B l_{\text {turb. numbers depend on }}$ the surface forces, which become dominant in the presence of nanoparticles and which cannot be neglected in a nanofluidic coolant, which was proved by us in Section 3.1 of this work. $B l_{\text {turb. }}$. number completely depends on the molecular $B l$ number due to the change in the degree of turbulence $(-X)$ of the refrigerant flow depending on external factors, for example, on temperature (Figure 4).

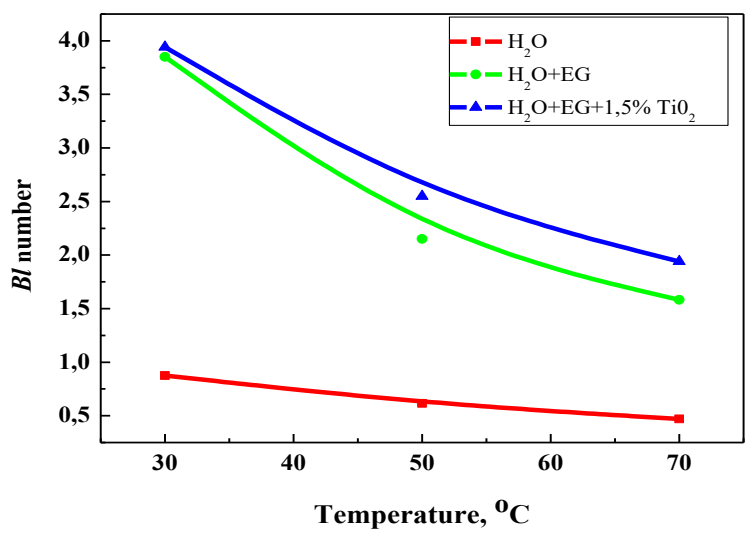

Figure 3. Dependence of the molecular $B l$ number on the coolant temperature

Molecular $\mathrm{Bl}$ number completely controls the turbulent $B l_{\text {turb }}$, number which confirms the conclusions of Italian scientists in the field of fluid mechanics [34]. Using the second equation, it is quite easy to analytically calculate the heat exchanger and evaluate the efficiency of a particular nanofluid in the heat exchanger, which we have done in the expressmethod according to formula (46) in section 3.6.2. of this work.

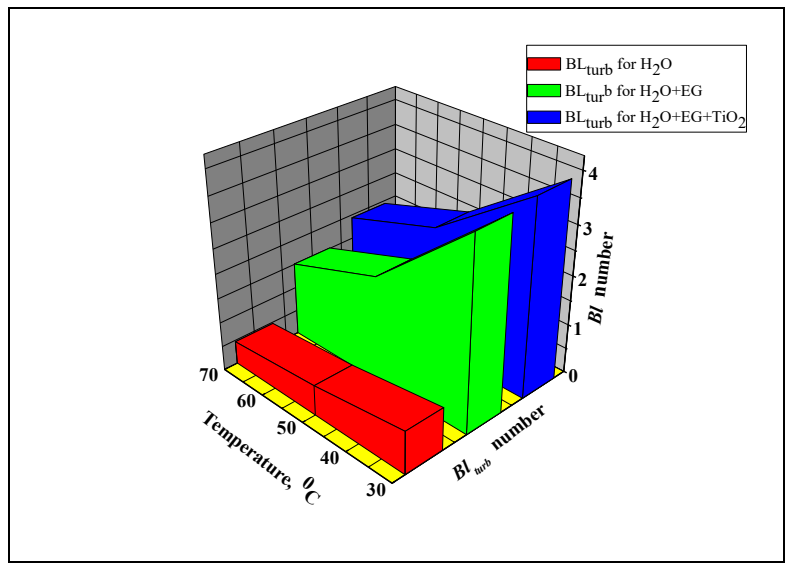

Figure 4. Influence of the molecular $B l$ number on the turbulent $B l_{\text {turb. }}$. number depending on temperature for various mixes of nanofluidic heat transfer fluids

We are convinced that when choosing a new generation coolant, which are nanofluids, one should use new generation numerical equations that take into account the action of surface forces. It should be remembered that it is the classical equations that serve as the foundation for new ones, which are based on the powerful experimental material of many generations of scientists.

Our approach, based on the classical works, does not contradict any of them. We are confident that formula (33), which is a new numerical equation for calculating heat exchangers with nanofluids, as well as formula (41 or 43) for analytical calculation of the degree of turbulence of the coolant flow $(-X)$, contain separate fragments of classical studies. Their combination, as well as taking into account the influence 
of surface forces during heat exchange with nanofluid heat carriers, allowed us corresponding result.

3.7 Algorithm for calculating a shell-and-tube heat exchanger using a new method for heating mix of (milk + $0.5 \%$ pumpkin oil) with hot mix of (water + EG (60:40) $+1.5 \% \mathrm{TiO}_{2}$ )

In our previous work [16], we calculated a plate heat exchanger using the classical and new method using model liquids of water and milk. This paper presents express calculations of a shell-and-tube heat exchanger using nanofluid mixtures of water with ethylene glycol using $1.5 \%$ $\mathrm{TiO}_{2}$ nanoparticles. To milk, as the second model liquid, $0.5 \%$ pumpkin seed oil was added as a SAS. Natural SAS additives to milk, which alter its thermophysical properties, were studied by us, for example, in our previous work [37].

We present the calculation of a shell-and-tube heat exchanger using a new method using EG (60:40)+1.5\% $\mathrm{TiO}_{2}$ as an additive to water. The overall heat transfer coefficient of this shell-and-tube heat exchanger without the use of nanofluids was $U=788,7 \mathrm{~W} \cdot \mathrm{m}^{-1} \cdot \mathrm{K}^{-1}[25,26]$.

The thermophysical properties of the mixes (hot water + EG $\left.(60: 40)+1.5 \% \mathrm{TiO}_{2}\right)$ and (cold milk $+0.5 \%$ pumpkin oil) are presented earlier in the section 3.6.2 (Tables 4 and 5). The final results are presented in Table 6.

$2 \cdot \frac{1}{3000}-$ thermal resistance of contaminants (Table 6);

$r_{c}=d_{c} / 2=21.10^{-3} / 2=10.5 \cdot 10^{-3} \mathrm{~m}$. (radius in the tube space of the shell-and-tube heat exchanger);

$r_{h}=d_{h} / 2=25 \cdot 10^{-3} / 2=12 \cdot 5 \cdot 10^{-3} \mathrm{~m}$. (equivalent radius of the annular space) (Table 6);

Increase in overall heat transfer coefficient compared to pure water and pure milk:

$$
\begin{gathered}
\Delta_{\text {water + EG (60:40)+1.5\% } \mathrm{TiO}_{2}}=\frac{920.81-788.7}{788.7} \cdot 100 \%=16.75 \% ; \\
\Delta_{\text {water + EG (60:40) }}=\frac{890.6-788.7}{788.7} \cdot 100 \%=12.86 \% ;
\end{gathered}
$$

\begin{tabular}{|c|c|c|}
\hline Parameter with formula and numbering & $\begin{array}{c}\text { Cold milk }+0.5 \% \text { pumpkin oil } \\
(\mathrm{c})\end{array}$ & $\begin{array}{l}\left.\text { Hot mix of (water + EG }(60: 40)+1.5 \% \mathrm{TiO}_{2}\right) \\
(\mathrm{h})\end{array}$ \\
\hline Molecular number $B l=\frac{\mu \sqrt{C_{p} \cdot 1 K^{0}}}{\sigma \cdot \cos \theta}$ & 1.756 & 1.941 \\
\hline $\begin{array}{l}\text { The average speed, } m \cdot s^{-1} \\
\text { The exponent }(-X) \\
-X=\frac{\operatorname{Re} \cdot \mu}{d \cdot \rho} \\
\ln \frac{a \cdot \sqrt{2 \operatorname{Re}}}{0.769 \cdot 1 / B l} \\
\ln \frac{\sqrt{C_{p} \cdot 1^{0} K}}{V}\end{array}$ & 0.659 & 0.690 \\
\hline 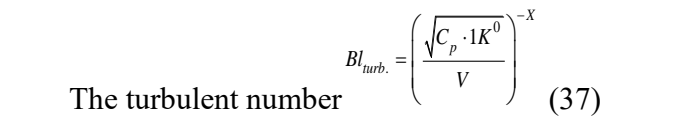 & 33.31 & 32.07 \\
\hline$k_{\text {turb. }}=(\sigma \cdot \cos \theta) \sqrt{C_{P} \cdot 1^{0} K}\left(\frac{\sqrt{C_{P} \cdot 1^{0} K}}{V}\right)$ & 66.38 & 84.24 \\
\hline $\begin{array}{l}\text { The overall heat transfer coefficient, W. } \mathrm{m}^{-2} \mathrm{~K}^{-1} \\
\qquad U_{\text {prop. }}=\left(\frac{r_{c}}{k_{\text {turb. } .}}+\frac{b_{w}}{k_{w}}+2 \frac{1}{3000}+\frac{r_{h}}{k_{\text {turb.h }}}\right)^{-1}\end{array}$ & & 920.81 \\
\hline
\end{tabular}

Table 6. Calculating a shell-and-tube heat exchanger using a new method

Note that the results of the full calculation of this shell-andtube heat exchanger completely coincide with the results of our new express method in section 3.6.2.

\section{CONCLUSION}

1. Classical numerical equations, which are widely used when choosing heat exchangers with nanofluids, give an error of $(15-20) \%$ or more. This leads to a partial waste of heat energy and a negative environmental situation.

2. A simulation of the motion of nanoparticles in a turbulent flow of a liquid coolant with allowance for surface forces has been carried out. The Froude, Reynolds, Euler numbers and the surface criterion in the polymolecular boundary layer (LBL) are calculated. It is shown that the surface forces are several orders of magnitude higher than the rest.
3. New formulas are obtained for a qualitative assessment of the average thickness of the LBL arising around a turbulently moving solid nanoparticle. A number of qualitative correlations are shown to explain the behavior of solid nanoparticles in a turbulent liquid medium.

4. A new approach to heat transfer processes using the values of turbulent viscosity and thermal conductivity of a liquid or gaseous coolant is considered.

5. Taking into account the MDM, new formulas (41 and 43) were obtained for the analytical calculation of the degree of turbulence of the coolant flow under the action of surface forces and Stokes resistance forces on nanoparticles.

6. Shown the physical meaning of the new similarity numbers $B l$ and $B l_{\text {turb., }}$ Obtained by us earlier [16, 17], and the possibility of their effective use for calculating heat exchangers with nanofluid coolants; 
7. A new express method for evaluating the efficiency of using nanofluids in heat exchange equipment is proposed, based on a new equation using the similarity numbers $B l$ and $B l_{\text {turb. }}$.

8. The proposed method of express calculation shows that the mixture $\mathrm{H}_{2} \mathrm{O}+\mathrm{EG}(60: 40)$ improves the heat exchange properties of water by $+12.86 \%$, and the mixtures $\left(\mathrm{H}_{2} \mathrm{O}+\mathrm{EG}\right.$ $\left.(60: 40)+1.5 \% \mathrm{TiO}_{2}\right)$ and $(+0.5 \%$ pumpkin seed oil $)-$ by + $16.75 \%$, which corresponds to experiments [36] and our computer simulation [16], and the well-known express method shows a deterioration by $-4.5 \%$ and, accordingly, by $-1.2 \%$;

9. An example of calculating a shell-and-tube heat exchanger when heating milk with hot water with the addition of mixtures $\left(\mathrm{H}_{2} \mathrm{O}+\mathrm{EG}(60: 40)+1.5 \% \mathrm{TiO}_{2}\right)$ and (milk $+0.5 \%$ pumpkin seed oil.) According to the new method fully confirms the effectiveness of the new express - method.

\section{REFERENCES}

[1] Reichardt, H. (1944). Impuls-und Wärmeaustausch in freier Turbulenz. ZAMM-Journal of Applied Mathematics and Mechanics/Zeitschrift für Angewandte Mathematik und Mechanik, 24(5-6): 268-272. https://doi.org/10.1002/zamm.19440240515

[2] Schlichting, H., Gersten, K. (2003). Boundary-layer theory. Nev York, USA: 1979. 706-707, 752-753, 741, $747,749,754$.

[3] Dytnerskij, Y.I. (1991). Basic processes and devices of chemical technology (Manual of engineering). Moscow, SU: Chemistry.

[4] Pak, B.C., Cho, Y.I. (1998). Hydrodynamic and heat transfer study of dispersed fluids with submicron metallic oxide particles. Experimental Heat Transfer an International Journal, 11(2): 151-170. https://doi.org/10.1080/08916159808946559

[5] Maïga, S.E.B., Nguyen, C.T., Galanis, N., Roy, G., Maré, T., Coqueux, M. (2006). Heat transfer enhancement in turbulent tube flow using $\mathrm{Al}_{2} \mathrm{O}_{3}$ nanoparticle suspension. International Journal of Numerical Methods for Heat \& Fluid Flow, 16(3): 275-292. https://doi.org/10.1108/09615530610649717.

[6] Petukhov, B.S. (1970). Heat transfer and friction in turbulent pipe flow with variable physical properties. In Advances in Heat Transfer, 6: 503-564. https://doi.org/10.1016/S0065-2717(08)70153-9

[7] Gnielinski, V. (1976). New equations for heat and mass transfer in turbulent pipe and channel flow. Int. Chem. Eng., 16(2): 359-368.

[8] Buongiorno, J. (2006). Convective transport in nanofluids. Journal of Heat Transfer, 128: 240-250. http://dx.doi.org/10.1115/1.2150834

[9] Xuan, Y., Li, Q. (2003). Investigation on convective heat transfer and flow features of nanofluids. J. Heat Transfer, 125(1): 151-155. http://dx.doi.org/10.1115/1.1532008

[10] Duangthongsuk, W., Wongwises, S. (2010). An experimental study on the heat transfer performance and pressure drop of $\mathrm{TiO}_{2}$-water nanofluids flowing under a turbulent flow regime. International Journal of Heat and Mass Transfer, 53(1-3): 334-344. https://doi.org/10.1016/j.ijheatmasstransfer.2009.09.024

[11] Vajjha, R.S., Das, D.K., Kulkarni, D.P. (2010). Development of new correlations for convective heat transfer and friction factor in turbulent regime for nanofluids. International Journal of Heat and Mass Transfer, 53(21-22): 4607-4618. http://dx.doi.org/10.1016/j.ijheatmasstransfer.2010.06.0 32

[12] Asirvatham, L.G., Raja, B., Lal, D.M., Wongwises, S. (2011). Convective heat transfer of nanofluids with correlations. Particuology, 9(6): 626-631. http://dx.doi.org/10.1016/j.partic.2011.03.014

[13] Elias, M.M., Rahman, S., Rahim, N.A., Sohel, M.R., Mahbubul, I.M. (2014). Performance investigation of a plate heat exchanger using nanofluid with different chevron angle. In Advanced Materials Research, 832: 254-259. 10.4028/www.scientific.net/AMR.832.254

[14] Elias, M.M., Mahbubul, I.M., Saidur, R., Sohel, M.R., Shahrul, I.M., Khaleduzzaman, S.S., Sadeghipour, S. (2014). Experimental investigation on the thermophysical properties of $\mathrm{Al}_{2} \mathrm{O}_{3}$ nanoparticles suspended in car radiator coolant. International Communications in Heat and Mass Transfer, 54: 48-53. https://doi.org/10.1016/j.icheatmasstransfer.2014.03.00 5

[15] Huang, D., Wu, Z., Sunden, B. (2016). Effects of hybrid nanofluid mixture in plate heat exchangers. Experimental Thermal and Fluid Science, 72: 190-196. https://doi.org/10.1016/j.expthermflusci.2015.11.009

[16] Bilonoga, Y., Stybel, V., Maksysko, O., Drachuk, U. (2020). A new universal numerical equation and a new method for calculating heat-exchange equipment using nanofluids. International Journal of Heat and Technology, 38(1): 151-164. https://doi.org/10.18280/ijht.380117

[17] Rudyak, V.Y., Minakov, A.V., Krasnolutskii, S.L. (2016). Physics and mechanics of heat exchange processes in nanofluid flows. Physical Mesomechanics, 19(3): 298-306. https://doi.org/10.1134/S1029959916030085

[18] Timofeeva, E.V., Yu, W., France, D.M., Singh, D., Routbort, J.L. (2011). Base fluid and temperature effects on the heat transfer characteristics of $\mathrm{SiC}$ in ethylene glycol/ $\mathrm{H}_{2} \mathrm{O}$ and $\mathrm{H}_{2} \mathrm{O}$ nanofluids. Journal of Applied Physics, $109(1)$ : 014914. https://doi.org/10.1063/1.3524274

[19] Patra, A.K., Nayak, M.K., Misra, A. (2020). Viscosity of nanofluids - A review. International Journal of Thermofluid Science and Technology, 7(2): 070202 https://doi.org/10.36963/IJTST.2020070202

[20] Rudyak, V.Y., Minakov, A.V. (2018). Thermophysical properties of nanofluids. Eur. Phys. J. E, 41(1): 1-12. https://doi.org/10.1140/epje/i2018-11616-9

[21] Rudyak, V.Y., Belkin, A. (2010). A. Modeling of transfer coefficients of nanofluids. Nanosystems: Physics, Chemistry, Mathematics, 1(1): 156-177.

[22] Rudyak, V.Y., Belkin, A.A., Krasnolutskii, S.L. (2005). Statistical theory of nanoparticle transport processes in gases and liquids. Thermophysics and Aeromechanics, 12(4): 489-507.

[23] Rudyak, V., Belkin, A., Tomilina, E. (2008). The force acting on a nanoparticle in a liquid. Technical Physics Letters, 34(1): 76-78. https://doi.org/10.1134/S1063785008010239

[24] Bilonoga, Y., Maksysko, O. (2017). Modeling the interaction of coolant flows at the liquid-solid boundary with allowance for the laminar boundary layer. International Journal of Heat and Technology, 35(3): 678-682. http://dx.doi.org/10.18280/ijht.350329 
[25] Bilonoga, Y., Maksysko, O. (2018). Specific features of heat exchangers calculation considering the laminar boundary layer, the transitional and turbulent thermal conductivity of heat carriers. International Journal of Heat and Technology, 36(1): 11-20. https://doi.org/10.18280/ijht.360102

[26] Bilonoga, Y., Maksysko, O. (2019). The laws of distribution of the values of turbulent thermo-physical characteristics in the volume of the flows of heat carriers taking into account the surface forces. International Journal of Heat and Technology, 37: 1-10. https://doi.org/10.18280/ijht.370101

[27] Mishra, S., Nayak, M., Misra, A. (2020). Thermal conductivity of nanofluids-A comprehensive review. International Journal of Thermofluid Science and Technology, 7(3): 070301. https://doi.org/10.36963/IJTST.2020070301

[28] Jiang, H., Xu, Q., Huang, C., Shi, L. (2015). The role of interfacial nanolayer in the enhanced thermal conductivity of carbon nanotube-based nanofluids. Applied Physics A, 118(1): 197-205. https://doi.org/10.1007/s00339-014-8902-5

[29] Pokhmurskii, V.I., Sirak, Y.M., Bilonoga, Y.L. (1984). Influence of the surface energy and of the energy of the bond of the contacting metals on the fretting fatigue life of the joints of machine parts. Soviet materials science: A transl. of Fiziko-khimicheskaya mekhanika materialov/Academy of Sciences of the Ukrainian SSR, 20(4): 358-360. https://doi.org/10.1007/BF01199367

[30] Bilonoga, Y.L., Pokhmurs' kii, V.I. (1991). A connection between the fretting-fatigue endurance of steels and the surface energy of the abradant metal. Soviet materials science: A transl. of Fiziko-khimicheskaya mekhanika materialov/Academy of Sciences of the Ukrainian SSR, 26(6): 629-633. https://doi.org/10.1007/BF00723647

[31] Pokhmurskii, V.I., Bilonoga, Y.L., Sirak, Y.M., German, N.V. (1986). Some principles of the development of a fretting-resistant lubricant. Soviet materials science: A transl. of Fiziko-khimicheskaya mekhanika materialov/Academy of Sciences of the Ukrainian SSR, 21(6): 593-595. https://doi.org/10.1007/BF00722252

[32] Oleshko, V., Pigovkin, I. (2016). Operational determination of surface energy of metal parts of aviation equipment. Internet-journal " NAUKOVEDENIE", 8(3): $1-15$.

[33] Bayareh, M. (2019). Numerical simulation and analysis of heat transfer for different geometries of corrugated tubes in a double pipe heat exchanger. Journal of Thermal Engineering, 5(4): 293-301. https://doi.org/10.18186/thermal.581775

[34] Quadrio, M., Ricco, P. (2011). The laminar generalized Stokes layer and turbulent drag reduction. Journal of Fluid Mechanics, 667: 135-157. http://dx.doi.org/10.1017/S0022112010004398

[35] Pavlenko, I., Sklabinskyi, V., Pitel', J., Židek, K., Kuric, I., Ivanov, V., Liaposhchenko, O. (2020). Effect of superimposed vibrations on droplet oscillation modes in prilling process. Processes, 8(5): 566. https://doi.org/10.3390/pr8050566

[36] Hamid, K.A., Azmi, W.H., Mamat, R., Usri, N.A., Najafi, G. (2015). Effect of temperature on heat transfer coefficient of titanium dioxide in ethylene glycol-based nanofluid. Journal of Mechanical Engineering and Sciences,

$1367-1375$ http://dx.doi.org/10.15282/jmes.8.2015.11.0133

[37] Bilonoga, Y., Stybel, V., Lorenzini, E., Maksysko, O., Drachuk, U. (2019). Changes in the hydro-mechanical and thermo-physical characteristics of liquid food products (for example, milk) under the influence of natural surfactants. Italian Journal of Engineering Science: Tecnica Italiana, 63(1): 21-27. https://doi.org/10.18280/ti-ijes.630103

[38] Chubik, I.A., Maslov, A.M. (1970). Handbook on the thermophysical characteristics of food products and semi-finished products. M: Food Industry, 184.

[39] MacDevette, M. (2014). Heat transfer analysis of nanofluids and phase change materials. Doctoral dissertation, Universitat Politècnica de Catalunya (UPC).

\section{NOMENCLATURE}

$\begin{array}{ll}a & \text { experimental coefficient } \\ b & \end{array}$

$b$ acceleration of inertia force, $\mathrm{m} . \mathrm{s}^{-2}$

$B$ dimensionless constant

$B l \quad$ dimensionless number

$B l_{\text {turb. }} \quad$ turbulent dimensionless number

$\cos \theta$ the hydrophilicity of the nanoparticle surface

$D \quad$ equivalent channel diameter, $\mathrm{m}$

d diameter, $\mathrm{m}$

$E \quad$ unknown exponents

$F_{\sigma} \quad$ force of surface tension of a liquid, N

$h \quad$ heat transfer coefficient, $\mathrm{W} \cdot \mathrm{m}^{-2} \cdot \mathrm{K}^{-1}$

$k \quad$ thermal conductivity, W. $\mathrm{m}^{-1} \cdot \mathrm{K}^{-1}$

$k_{\text {turb }} \quad$ coefficient of average turbulent thermal conductivity, W. $\mathrm{m}^{-1} \cdot \mathrm{K}^{-1}$

$k_{w} \quad$ thermal conductivity of stainless steel, $\mathrm{W} \cdot \mathrm{m}^{-1} \cdot \mathrm{K}^{-1}$

$M \quad$ unknown exponents

$N \quad$ modified Reynolds number in LBL

$P \quad$ unknown exponents

$\mathrm{Nu} \quad$ Nusselt number

$\mathrm{Pr} \quad$ Prandtl number

$r \quad$ pipe radius, $\mathrm{m}$

$R \quad$ radius, $\mathrm{m}$

Re Reynolds number

$S \quad$ unknown exponents

$t \quad$ temperature, ${ }^{\circ} \mathrm{C}$

$U \quad$ overall heat transfer coefficient, W. $\mathrm{m}^{-2} \cdot \mathrm{K}^{-1}$

$V \quad$ velocity, $\mathrm{m} . \mathrm{s}^{-1}$

$W \quad$ volume flow rate of liquid (coolant), $\mathrm{m}^{3} \cdot \mathrm{s}^{-1}$

$X \quad$ unknown exponents

$Y \quad$ unknown exponents

\section{Greek symbols}

$\delta \quad$ average thickness LBL, $\mathrm{m}$

$\mu \quad$ coefficient viscosity of coolant, $\mathrm{kg} \cdot \mathrm{m}^{-1} \cdot \mathrm{s}^{-1}$

$\mu_{\text {turb }} \quad$ coefficient of turbulent viscosity of coolant, $\mathrm{kg} \cdot \mathrm{m}^{-1} \cdot \mathrm{s}^{-1}$

$\rho \quad$ fluid density, $\mathrm{kg} \cdot \mathrm{m}^{-3}$

$\rho_{n f} \quad$ density of nanofluid, $\mathrm{kg} \cdot \mathrm{m}^{-3}$

$\sigma \quad$ surface tension coefficient of coolant, N.m ${ }^{-1}$

\section{Subscripts}

c $\quad$ cold (milk)

$f \quad$ fluid 
$h \quad$ hot (water)

$L \quad$ Laminar regime

nf nanofluid

$T \quad$ turbulent regime

trans transitional

turb turbulent

w wall

\section{Abbreviations}

EG ethylene glycol

$L B L \quad$ laminar boundary layer

MDM molecular dynamics method

PMBLLM poly-molecular boundary layer of liquid molecules 